\title{
HARMONIC ANALYSIS ON THE EINSTEIN GYROGROUP
}

\section{MILTON FERREIRA}

Communicated by Abraham A. Ungar

Abstract. In this paper we study harmonic analysis on the Einstein gyrogroup of the open ball of $\mathbb{R}^{n}, n \in \mathbb{N}$, centered at the origin and with arbitrary radius $t \in \mathbb{R}^{+}$, associated to the generalised Laplace-Beltrami operator

$$
L_{\sigma, t}=\left(1-\frac{\|x\|^{2}}{t^{2}}\right)\left(\Delta-\sum_{i, j=1}^{n} \frac{x_{i} x_{j}}{t^{2}} \frac{\partial^{2}}{\partial x_{i} \partial x_{j}}-\frac{\kappa}{t^{2}} \sum_{i=1}^{n} x_{i} \frac{\partial}{\partial x_{i}}+\frac{\kappa(2-\kappa)}{4 t^{2}}\right)
$$

where $\kappa=n+\sigma$ and $\sigma \in \mathbb{R}$ is an arbitrary parameter. The generalised harmonic analysis for $L_{\sigma, t}$ gives rise to the $(\sigma, t)$-translation, the $(\sigma, t)$-convolution, the $(\sigma, t)$-spherical Fourier transform, the $(\sigma, t)$-Poisson transform, the $(\sigma, t)$-Helgason Fourier transform, its inverse transform and Plancherel's Theorem. In the limit of large $t, t \rightarrow+\infty$, the resulting hyperbolic harmonic analysis tends to the standard Euclidean harmonic analysis on $\mathbb{R}^{n}$, thus unifying hyperbolic and Euclidean harmonic analysis.

\section{Contents}

3 The $(\sigma, t)$-Translation

4 The $(\sigma, t)$-Convolution

5 Eigenfunctions of $L_{\sigma, t}$

6 The $(\sigma, t)$-Poisson transform

7 The $(\sigma, t)$-Helgason Fourier Transform

8 Inversion of the $(\sigma, t)$-Helgason Fourier Transform and Plancherel's Theorem 50

9 Appendices 
A Spherical Harmonics

B Jacobi Functions

56

References

\section{Introduction}

In this paper we are interested in developing harmonic analysis on the KleinBeltrami ball model of hyperbolic geometry, a model regulated algebraically by the Einstein addition law of relativistically admissible velocities. Indeed, Einstein addition plays a role similar to that of vector addition in the Euclidean $n$-space $\mathbb{R}^{n}$ giving rise to the Einstein gyrogroup $[14,15,18]$. The gyrogroup structure is a natural extension of the group structure, discovered in 1988 by A. Ungar [14] in the context of Einstein's velocity addition law. Einstein addition corresponds to the standard velocity addition of relativistically admissible velocities that Einstein introduced in his 1905 paper that founded the special theory of relativity. The Einstein gyrogroup resolves the breakdown of associativity and commutativity introducing the gyration operator which corresponds to the Thomas precession rotation. In this way, the Thomas precession rotation is studied in the algebraic structure of the ball and not as an isolated phenomenon. Several new results were discovered applying gyrogroup theoretic techniques, as can be seen in the works of A. Ungar and others [4, 6, 16, 18-24].

In this paper we study several aspects of harmonic analysis on the Einstein gyrogroup $\left(\mathbb{B}_{t}^{n}, \oplus\right), n \in \mathbb{N}$, associated to the family of Laplace-Beltrami operators $L_{\sigma, t}$ given by

$$
L_{\sigma, t}=\left(1-\frac{\|x\|^{2}}{t^{2}}\right)\left(\Delta-\sum_{i, j=1}^{n} \frac{x_{i} x_{j}}{t^{2}} \frac{\partial^{2}}{\partial x_{i} \partial x_{j}}-\frac{\kappa}{t^{2}} \sum_{i=1}^{n} x_{i} \frac{\partial}{\partial x_{i}}+\frac{\kappa(2-\kappa)}{4 t^{2}}\right)
$$

where $\kappa=n+\sigma$, with $\sigma \in \mathbb{R}$, and $t \in \mathbb{R}^{+}$is the radius of the ball $\mathbb{B}_{t}^{n}=$ $\left\{x \in \mathbb{R}^{n} ;\|x\|<t\right\}$. The operator $L_{\sigma, t}$ is a generalisation of the Laplace-Beltrami operator $\widetilde{L}$ defined on the Klein model of the unit ball

$$
\widetilde{L}=\left(1-\|x\|^{2}\right)\left(\Delta-\sum_{i, j=1}^{n} x_{i} x_{j} \frac{\partial^{2}}{\partial x_{i} \partial x_{j}}-2 \sum_{i=1}^{n} x_{i} \frac{\partial}{\partial x_{i}}\right)
$$

considered e.g. in [25]. For the special case when $\sigma=2-n$ and $t=1$ we have $L_{2-n, 1}=\widetilde{L}$. The operators $L_{\sigma, 1}$ can also be seen as the real counterparts of the 
$(\alpha, \beta)$-Laplacians $\Delta_{\alpha, \beta}$ on the unit complex ball given by

$\Delta_{\alpha, \beta}=4\left(1-\|z\|^{2}\right)\left(\sum_{i, j=1}^{n}\left(\delta_{i j}-z_{i} \bar{z}_{j}\right) \frac{\partial^{2}}{\partial z_{i} \partial \bar{z}_{j}}+\alpha \sum_{j=1}^{n} z_{j} \frac{\partial}{\partial z_{j}}+\beta \sum_{j=1}^{n} \bar{z}_{j} \frac{\partial}{\partial \bar{z}_{j}}-\alpha \beta\right)$.

These operators were studied in several papers, e.g. [2,26] where the authors established a weighted Plancherel formula and characterised the $L^{2}$-range of the Poisson transform associated to the $(\alpha, \beta)$-Laplacians $\Delta_{\alpha, \beta}$, respectively.

Using the gyrolanguage we study several aspects of gyroharmonic analysis on the Einstein gyrogroup depending on an arbitrary parameter $\sigma \in \mathbb{R}$ and the radius $t \in \mathbb{R}^{+}$of the ball $\mathbb{B}_{t}^{n}$. It includes the $(\sigma, t)$-translation, the $(\sigma, t)$-convolution, eigenfunctions of $L_{\sigma, t},(\sigma, t)$-spherical functions, the $(\sigma, t)$-Poisson transform, the $(\sigma, t)$-Helgason Fourier transform, its inverse and Plancherel's Theorem. While the general theory about Fourier transform on hyperbolic space can be seen in the works of Helgason $[8,9]$ in this paper we obtain new results like a Young's inequality for the $(\sigma, t)$-convolution (Theorem 12), the gyrotranslation invariance of the $(\sigma, t)$-convolution (Theorem 15), the gyroassociative law of the $(\sigma, t)$-convolution (Theorem 16), and the gyroconvolution theorem with respect to the $(\sigma, t)$-Helgason Fourier transform (Theorem 35). Remarkably, each of these theorems involves the gyration operator.

This work is in the same spirit of our previous work [5] where we developed harmonic analysis on the Möbius gyrogroup. The results of this paper can be applied in different settings, e.g. wavelet analysis, where the information about Fourier coefficients is important for the definition of a continuous wavelet transform on the ball. It is also possible to construct coherent states for $L^{2}$-eigenspaces associated to the discrete spectrum of the Schrödinger operator with uniform magnetic field on $\mathbb{B}_{t}^{n}$ as was done in [3] for the case of the unit complex ball. In all these areas harmonic analysis is connected with representation theory. A nice overview of the relationship between harmonic analysis and quantum mechanics can be seen for instance in [13].

The paper is organised as follows. In Section 2 we present the Einstein addition in $\mathbb{B}_{t}^{n}$ and its properties. Sections 3 and 4 are dedicated to the study of the $(\sigma, t)$ translation and the $(\sigma, t)$-convolution. In Section 5 we construct the eigenfunctions of the generalised Laplace-Beltrami operator on the Einstein gyrogroup and we study the associated $(\sigma, t)$-spherical functions. In Section 6 we define the $(\sigma, t)$ Poisson transform and study the injectivity of this transform. Section 7 is devoted to the $(\sigma, t)$-Helgason Fourier transform, which is the relativistic counterpart of the Euclidean Fourier transform. In Section 8 we obtain the inversion formula for the $(\sigma, t)$-Helgason Fourier transform, the Plancherel's Theorem, and show that 
in the limit $t \rightarrow+\infty$ we recover the inverse Fourier transform and Plancherel's Theorem in Euclidean harmonic analysis. Two Appendices, A and B, concerning all necessary facts on spherical harmonics and Jacobi functions, are found at the end of the paper.

\section{Einstein Addition in the Ball}

The Beltrami-Klein model of the $n$-dimensional real hyperbolic geometry can be realised as the open ball $\mathbb{B}_{t}^{n}=\left\{x \in \mathbb{R}^{n} ;\|x\|<t\right\}$ of $\mathbb{R}^{n}$, endowed with the Riemannian metric

$$
\mathrm{d} s^{2}=\frac{\|\mathrm{d} x\|^{2}}{1-\frac{\|x\|^{2}}{t^{2}}}+\frac{(\langle x, \mathrm{~d} x\rangle)^{2}}{t^{2}\left(1-\frac{\|x\|^{2}}{t^{2}}\right)} .
$$

This metric corresponds to the metric tensor

$$
g_{i j}(x)=\frac{\delta_{i, j}}{1-\frac{\|x\|^{2}}{t^{2}}}+\frac{x_{i} x_{j}}{t^{2}\left(1-\frac{\|x\|^{2}}{t^{2}}\right)^{2}}, \quad i, j \in\{1, \ldots, n\}
$$

and its inverse is given by

$$
g^{i j}(x)=\left(1-\frac{\|x\|^{2}}{t^{2}}\right)\left(\delta_{i, j}-\frac{x_{i} x_{j}}{t^{2}}\right), \quad i, j \in\{1, \ldots, n\} .
$$

The group of all isometries of the Klein model consists of the elements of the group $\mathrm{O}(n)$ and the mappings given by

$$
T_{a}(x)=\frac{a+P_{a}(x)+\mu_{a} Q_{a}(x)}{1+\frac{1}{t^{2}}\langle a, x\rangle}
$$

where

$$
P_{a}(x)=\left\{\begin{array}{ll}
\langle a, x\rangle \frac{a}{\|a\|^{2}} & \text { if } a \neq 0 \\
0 & \text { if } a=0
\end{array}, \quad Q_{a}(x)=x-P_{a}(x), \quad \text { and } \mu_{a}=\sqrt{1-\frac{\|a\|^{2}}{t^{2}}} .\right.
$$

Some properties are listed in the next Proposition.

Proposition 1. Let $a \in \mathbb{B}_{t}^{n}$. Then

i) $P_{a}^{2}=P_{a}, \quad Q_{a}^{2}=Q_{a}, \quad\left\langle a, P_{a}(x)\right\rangle=\langle a, x\rangle, \quad$ and $\quad\left\langle a, Q_{a}(x)\right\rangle=0$.

ii) $T_{a}(0)=a$ and $T_{a}(-a)=0$. 
iii) $T_{a}\left(T_{-a}(x)\right)=T_{-a}\left(T_{a}(x)\right)=x, \quad x \in \mathbb{B}_{t}^{n}$.

iv) $T_{a}\left( \pm t \frac{a}{\|a\|}\right)= \pm t \frac{a}{\|a\|}$. Moreover, the mapping $T_{a}$ fixes two points on $\partial \mathbb{B}_{t}^{n}$ and no point of $\mathbb{B}_{t}^{n}$.

v) The identity

$$
1-\frac{\left\langle T_{a}(x), T_{a}(y)\right\rangle}{t^{2}}=\frac{\left(1-\frac{\|a\|^{2}}{t^{2}}\right)\left(1-\frac{\langle x, y\rangle}{t^{2}}\right)}{\left(1+\frac{\langle x, a\rangle}{t^{2}}\right)\left(1+\frac{\langle y, a\rangle}{t^{2}}\right)}
$$

holds for all $x, y \in \mathbb{B}_{t}^{n}$. In particular, when $x=y$ we have

$$
1-\frac{\left\|T_{a}(x)\right\|^{2}}{t^{2}}=\frac{\left(1-\frac{\|a\|^{2}}{t^{2}}\right)\left(1-\frac{\|x\|^{2}}{t^{2}}\right)}{\left(1+\frac{\langle x, a\rangle}{t^{2}}\right)^{2}}
$$

and in (2) when $x=0$ we obtain

$$
1-\frac{\left\langle a, T_{a}(y)\right\rangle}{t^{2}}=\frac{1-\frac{\|a\|^{2}}{t^{2}}}{1+\frac{\langle y, a\rangle}{t^{2}}}
$$

vi) For $R \in O(n)$

$$
R \circ T_{a}=T_{R a} \circ R .
$$

The Laplace-Beltrami operator which commutes with the mappings $T_{a}(x)$ and the group $\mathrm{O}(n)$ is the operator

$$
L=\left(1-\frac{\|x\|^{2}}{t^{2}}\right)\left(\Delta-\sum_{i, j=1}^{n} \frac{x_{i} x_{j}}{t^{2}} \frac{\partial^{2}}{\partial x_{i} \partial x_{j}}+\frac{2}{t^{2}} \sum_{i=1}^{n} x_{i} \frac{\partial}{\partial x_{i}}\right)
$$

This operator was considered in [25] for the case of the unit ball with a change sign in the last term due to the use of the mappings $T_{a}(-x)$ instead of $T_{a}(x)$. There is a major difference between both mappings since the first is an involution while the second is not an involution. Moreover, the mappings $T_{a}(x)$ give rise to a gyrogroup structure while the mappings $T_{a}(-x)$ do not give rise.

To endow the ball $\mathbb{B}_{t}^{n}$ with a binary operation, closely related to vector addition in $\mathbb{R}^{n}$, we define the Einstein addition on $\mathbb{B}_{t}^{n}$ by

$$
a \oplus x:=T_{a}(x), \quad a, x \in \mathbb{B}_{t}^{n} .
$$


The algebraic structure $\left(\mathbb{B}_{t}^{n}, \oplus\right)$ is a gyrogroup (see [18]) with identity 0 , the inverse of $a \in \mathbb{B}_{t}^{n}$ is $\ominus a=-a$, and the binary operation $\oplus$ is left gyroassociative, i.e.,

$$
a \oplus(b \oplus c)=(a \oplus b) \oplus \operatorname{gyr}[a, b] c, \quad a, b, c \in \mathbb{B}_{t}^{n}
$$

where the map gyr $[a, b]: \mathbb{B}_{t}^{n} \rightarrow \mathbb{B}_{t}^{n}$ belongs to the automorphism group of $\mathbb{B}_{t}^{n}$ and satisfies the left loop property

$$
\operatorname{gyr}[a, b]=\operatorname{gyr}[a \oplus b, b] .
$$

The gyration operator also known as Thomas gyration can be given in terms of the Einstein addition $\oplus$ by the equation (see [18])

$$
\operatorname{gyr}[a, b] c=\ominus(a \oplus b) \oplus(a \oplus(b \oplus c)) .
$$

The Einstein gyrogroup is gyrocommutative since Einstein addition satisfies

$$
a \oplus b=\operatorname{gyr}[a, b](b \oplus a) .
$$

In the limit $t \rightarrow+\infty$, the ball $\mathbb{B}_{t}^{n}$ expands to the whole of the space $\mathbb{R}^{n}$, Einstein addition reduces to vector addition in $\mathbb{R}^{n}$ and, therefore, the gyrogroup $\left(\mathbb{B}_{t}^{n}, \oplus\right)$ reduces to the translation group $\left(\mathbb{R}^{n},+\right)$. Some useful gyrogroup identities ([18], pp. 48 and 68) that will be used in this paper are

$$
\begin{gathered}
\ominus(a \oplus b)=(\ominus a) \oplus(\ominus b) \\
a \oplus(\ominus a \oplus b)=b \\
\operatorname{gyr}[a, b])^{-1}=\operatorname{gyr}[b, a] \\
\operatorname{gyr}[a \oplus b, \ominus a]=\operatorname{gyr}[a, b] \\
\operatorname{gyr}[\ominus a, \ominus b]=\operatorname{gyr}[a, b] \\
\operatorname{gyr}[a, \ominus a]=I \\
\operatorname{gyr}[a, b](b \oplus(a \oplus c))=(a \oplus b) \oplus c .
\end{gathered}
$$

Properties (13) and (14) are valid for general gyrogroups while properties (11) and (17) are valid only for gyrocommutative gyrogroups. Combining formulas (14) and (17) with (13) we obtain the identities

$$
\begin{gathered}
\operatorname{gyr}[\ominus a, a \oplus b]=\operatorname{gyr}[b, a] \\
b \oplus(a \oplus c)=\operatorname{gyr}[b, a]((a \oplus b) \oplus c) .
\end{gathered}
$$

In the special case when $n=1$, the Einstein gyrogroup becomes a group since gyrations are trivial (a trivial map being the identity map). For $n \geq 2$ the gyrosemidirect product of $\left(\mathbb{B}_{t}^{n}, \oplus\right)$ and $\mathrm{O}(n)$ (see [18]) gives the group $\mathbb{B}_{t}^{n} \rtimes_{\text {gyr }} \mathrm{O}(n)$ for the operation

$$
(a, R)(b, S)=(a \oplus R b, \operatorname{gyr}[a, R b] R S) .
$$

This group is a realisation of the Lorentz group $\mathrm{O}(1, n)$. In the limit $t \rightarrow+\infty$ the group $\mathbb{B}_{t}^{n} \rtimes_{\text {gyr }} \mathrm{O}(n)$ reduces to the Euclidean group $\mathrm{E}(n)=\mathbb{R}^{n} \rtimes \mathrm{O}(n)$. 


\section{The $(\sigma, t)$-Translation}

Definition 2. For a function $f$ defined on $B_{t}^{n}, a \in \mathbb{B}_{t}^{n}$ and $\sigma \in \mathbb{R}$ we define the $(\sigma, t)$-translation $\tau_{a} f$ of $f$ by

$$
\tau_{a} f(x)=f((-a) \oplus x) j_{a}(x)
$$

with

$$
j_{a}(x)=\left(\frac{\sqrt{1-\frac{\|a\|^{2}}{t^{2}}}}{1-\frac{\langle a, x\rangle}{t^{2}}}\right)^{\frac{n+\sigma-2}{2}} .
$$

The multiplicative factor $j_{a}(x)$ agrees with the Jacobian of the transformation $T_{-a}(x)=(-a) \oplus x$ when $\sigma=n+4$. Thus, it stands for a generalisation of the Jacobian. In the case $\sigma=2-n$ the $(\sigma, t)$-translation reduces to $\tau_{a} f(x)=f((-a) \oplus x)$. Moreover, for any $\sigma \in \mathbb{R}$, we obtain in the limit $t \rightarrow+\infty$ the Euclidean translation operator $\tau_{a} f(x)=f(-a+x)=f(x-a)$.

Lemma 3. For any $a, b, x, y \in \mathbb{B}_{t}^{n}$ the following relations hold

$$
\begin{aligned}
& \text { i) } j_{-a}(-x)=j_{a}(x) \\
& \text { ii) } j_{a}(a) j_{a}(0)=1 \\
& \text { iii) } j_{a}(x)=j_{x}(a) j_{a}(0) j_{x}(x) \\
& \text { iv) } j_{a}(a \oplus x)=\left(j_{-a}(x)\right)^{-1} \\
& \text { v) } j_{(-a) \oplus x}(0)=j_{x \oplus(-a)}(0)=j_{x}(a) j_{a}(0)=j_{a}(x) j_{x}(0) \\
& \text { vi) } j_{(-a) \oplus x}((-a) \oplus x)=\left(j_{a}(x)\right)^{-1} j_{x}(x) \\
& \text { vii) } \tau_{a} j_{y}(x)=\left[\tau_{-a} j_{x}(y)\right] j_{x}(x) j_{y}(0) \\
& \text { viii) } \tau_{-a} j_{a}(x)=1 \\
& \text { ix) } \tau_{a} j_{y}(x)=j_{a \oplus y}(x) \\
& \text { x) } \tau_{a} f(x)=\left[\tau_{x} f(-\operatorname{gyr}[x, a] a)\right] j_{a}(0) j_{x}(x) \\
& \text { xi) } \tau_{b} \tau_{a} f(x)=\tau_{b \oplus a} f(\operatorname{gyr}[a, b] x) \\
& \text { xii) } \tau_{-a} \tau_{a} f(x)=f(x) \\
& \text { xiii) } \tau_{b} \tau_{a} f(x)=\left[\tau_{-b} \tau_{x} f(-\operatorname{gyr}[-b, x \oplus a] \operatorname{gyr}[x, a] a)\right] j_{a}(0) j_{x}(x) .
\end{aligned}
$$

Proof: Identities (22)-(24) can be easily checked by direct calculations. Equalities (25) and (26) follow from (4) and (3) respectively. Equality (27) follows from (23) and (26) since we have

$$
j_{(-a) \oplus x}((-a) \oplus x)=\left(j_{(-a) \oplus x}(0)\right)^{-1}=\left(j_{a}(x) j_{x}(0)\right)^{-1}=\left(j_{a}(x)\right)^{-1} j_{x}(x) .
$$


To prove equality (28) we note first that by (3) we can write $j_{a}(x)$ as

$$
j_{a}(x)=\left(\frac{1-\frac{\left\|T_{a}(-x)\right\|^{2}}{t^{2}}}{1-\frac{\|x\|^{2}}{t^{2}}}\right)^{\frac{n+\sigma-2}{4}} .
$$

By definition we have

$$
\tau_{a} j_{y}(x)=j_{y}((-a) \oplus x) j_{a}(x)=j_{y}\left(T_{-a}(x)\right) j_{a}(x) .
$$

Since

$$
\begin{aligned}
T_{y}\left(-T_{-a}(x)\right) & =y \oplus(-((-a) \oplus x)) & & \text { by } \\
& =y \oplus(a \oplus(-x)) & & \text { by } \\
& =\operatorname{gyr}[y, a]((a \oplus y) \oplus(-x)) & & \text { by } \\
& =\operatorname{gyr}[y, a] T_{a \oplus y}(-x) & & \text { by }
\end{aligned}
$$

then $\left\|T_{y}\left(-T_{-a}(x)\right)\right\|=\left\|T_{a \oplus y}(-x)\right\|$ and by (35) and (3) we have

$$
\begin{aligned}
j_{y}\left(T_{-a}(x)\right) & =\left(\frac{1-\frac{\left\|T_{a \oplus y}(-x)\right\|^{2}}{t^{2}}}{1-\frac{\left\|T_{-a}(x)\right\|^{2}}{t^{2}}}\right)^{\frac{n+\sigma-2}{4}} \\
& =\left(\frac{\left(1-\frac{\|a \oplus y\|^{2}}{t^{2}}\right)\left(1-\frac{\|x\|^{2}}{t^{2}}\right)}{\left(1-\frac{\langle x, a \oplus y\rangle}{t^{2}}\right)^{2}\left(1-\frac{\left\|T_{-a}(x)\right\|^{2}}{t^{2}}\right)}\right)^{\frac{n+\sigma-2}{4}} \\
& =\left(\frac{\left(1-\frac{\|a\|^{2}}{t^{2}}\right)\left(1-\frac{\|y\|^{2}}{t^{2}}\right)\left(1-\frac{\|x\|^{2}}{t^{2}}\right)}{\left(1+\frac{\langle a, y\rangle}{t^{2}}\right)^{2}\left(1-\frac{\langle x, a \oplus y\rangle}{t^{2}}\right)^{2}\left(1-\frac{\left\|T_{-a}(x)\right\|^{2}}{t^{2}}\right)}\right)^{\frac{n+\sigma-2}{4}} .
\end{aligned}
$$

Therefore, by (37) and (29) we obtain

$\tau_{a} j_{y}(x)=j_{y}\left(T_{-a}(x)\right) j_{a}(x)=j_{x}(a \oplus y) j_{-a}(y) j_{x}(x) j_{y}(0)=\left[\tau_{-a} j_{x}(y)\right] j_{x}(x) j_{y}(0)$.

Equality (29) follows from (25)

$$
\tau_{-a} j_{a}(x)=j_{a}(a \oplus x) j_{-a}(x)=\left(j_{-a}(x)\right)^{-1} j_{-a}(x)=1 .
$$

Equality (30) follows from (36) and (35)

$$
\tau_{a} j_{y}(x)=j_{y}\left(T_{-a}(x)\right) j_{a}(x)=j_{a \oplus y}(x) .
$$


To prove (31) we have the following identities

$$
\begin{array}{rlrl}
{\left[\tau_{x} f(-\operatorname{gy}[x, a] a)\right] j_{a}(0) j_{x}(x)} & & \\
= & f(-\operatorname{gyr}[x,(-x) \oplus a]((-x) \oplus a)) & & \\
& \times j_{x}(a) j_{a}(0) j_{x}(x) & & \text { by }(20) \\
= & f(-\operatorname{gyr}[a,-x]((-x) \oplus a)) j_{a}(x) & & \text { by }(18), \\
= & f(-(a \oplus(-x))) j_{a}(x) & & \text { by }(10) \\
= & f((-a) \oplus x) j_{a}(x) & & \text { by }(11) \\
= & \tau_{a} f(x) & & \text { by }(20) .
\end{array}
$$

Now we prove equality (32)

$$
\begin{aligned}
\tau_{b} \tau_{a} f(x) & =f((-a) \oplus((-b) \oplus x)) \tau_{b} j_{a}(x) & & \text { by }(20) \\
& =f(\operatorname{gyr}[-a,-b](((-b) \oplus(-a)) \oplus x)) j_{b \oplus a}(x) & & \text { by }(19),(30) \\
& =f(\operatorname{gyr}[a, b]((-(b \oplus a)) \oplus x)) j_{b \oplus a}(x) & & \text { by }(15),(11) \\
& =\tau_{b \oplus a} f(\operatorname{gyr}[a, b] x) & & \text { by }(20) .
\end{aligned}
$$

Equality (33) follows from (32) considering $b=-a$ since $(-a) \oplus a=0$ and gyr $[a,-a]=I$ by (16). Finally, we prove the last identity

$$
\begin{array}{rlr}
{\left[\tau_{-b} \tau_{x} f(-\operatorname{gyr}[-b, x \oplus a] \operatorname{gyr}[x, a] a)\right] j_{a}(0) j_{x}(x)} & \\
= & \left.\left[\tau_{-b} f(-\operatorname{gyr}[-b, x \oplus((-x) \oplus a))] \operatorname{gyr}[x,(-x) \oplus a]((-x) \oplus a)\right) j_{x} a\right] \\
& \times j_{a}(0) j_{x}(x) & \\
= & {\left[\tau_{-b} f(-\operatorname{gyr}[-b, a] \operatorname{gyr}[x,(-x) \oplus a]((-x) \oplus a)) j_{x} a\right] j_{a}(0) j_{x}(x)} \\
= & {[f(-\operatorname{gyr}[-b, b \oplus a] \operatorname{gyr}[x,(-x) \oplus(b \oplus a)]((-x) \oplus(b \oplus a)))} \\
& \left.\times\left(\tau_{-b} j_{x}(a)\right)\right] j_{a}(0) j_{x}(x) & \text { by }(20) \\
= & f(-\operatorname{gyr}[a, b] \operatorname{gyr}[b \oplus a,-x]((-x) \oplus(b \oplus a))) \tau_{b} j_{a}(x) & \text { by }(18),(28) \\
= & f(-\operatorname{gyr}[a, b]((b \oplus a) \oplus(-x))) \tau_{b} j_{a}(x) & \text { by }(10) \\
= & f(-(a \oplus(b \oplus(-x)))) \tau_{b} j_{a}(x) & \text { by }(19) \\
= & f((-a) \oplus((-b) \oplus x)) \tau_{b} j_{a}(x) & \text { by }(11) \\
= & \tau_{b} f((-a) \oplus x) j_{a}(x) & \text { by }(20) \\
= & \tau_{b} \tau_{a} f(x) & \text { by }(20) .
\end{array}
$$

Before we prove that the generalised Laplace-Beltrami operator $L_{\sigma, t}$ commutes with $(\sigma, t)$-translations we present a representation formula for the operator $L_{\sigma, t}$ using the Laplace operator in $\mathbb{R}^{n}$. 
Proposition 4. For each $f \in C^{2}\left(\mathbb{B}_{t}^{n}\right)$ and $a \in \mathbb{B}_{t}^{n}$

$$
\left(L_{\sigma, t} f\right)(a)=\left(j_{a}(0)\right)^{-1} \Delta\left(\tau_{-a} f\right)(0)+\frac{(2-n-\sigma)(n+\sigma)}{4 t^{2}} f(a) .
$$

Proof: Let $a \in \mathbb{B}_{t}^{n}$ and $R \in \mathrm{O}(n)$ such that $R a=\|a\| e_{1}$ with $e_{1}=(1,0, \ldots, 0) \in$ $\mathbb{R}^{n}$. Denote by $T_{1}, \ldots, T_{n}$ the coordinates of the mapping $T_{\|a\| e_{1}}$. Then by the chain rule we have

$$
\begin{aligned}
& \Delta\left(\tau_{-\|a\| e_{1}} f\right)(0)=\left[\sum_{j, k=1}^{n} \frac{\partial^{2} f}{\partial x_{j} \partial x_{k}}\left(\|a\| e_{1}\right) \sum_{i=1}^{n} \frac{\partial T_{k}}{\partial x_{i}}(0) \frac{\partial T_{j}}{\partial x_{i}}(0)\right. \\
& \left.+\sum_{k=1}^{n} \frac{\partial f}{\partial x_{k}}\left(\|a\| e_{1}\right) \sum_{i=1}^{n} \frac{\partial^{2} T_{k}}{\partial x_{i}^{2}}(0)\right] j_{-\|a\| e_{1}}(0) \\
& +2 \sum_{k=1}^{n} \frac{\partial f}{\partial x_{k}}\left(\|a\| e_{1}\right) \sum_{i=1}^{n} \frac{\partial T_{k}}{\partial x_{i}}(0) \frac{\partial j_{-\|a\| e_{1}}}{\partial x_{i}}(0)+f\left(\|a\| e_{1}\right) \sum_{i=1}^{n} \frac{\partial^{2} j_{-\|a\| e_{1}}}{\partial x_{i}^{2}}(0) .
\end{aligned}
$$

Since $T_{1}(x)=\frac{\|a\|+x_{1}}{1+\frac{\|a\| x_{1}}{t^{2}}} \quad$ and $\quad T_{k}(x)=\frac{\mu_{a} x_{k}}{1+\frac{\|a\| x_{1}}{t^{2}}}, k \in\{2, \ldots, n\}$ then

$$
\begin{aligned}
\frac{\partial T_{1}}{\partial x_{i}}(0) & =\mu_{a}^{2} \delta_{i, 1} & \frac{\partial T_{k}}{\partial x_{i}}(0) & =\mu_{a} \delta_{k, i} \\
\frac{\partial^{2} T_{1}}{\partial x_{i}^{2}}(0) & =-2 \frac{\|a\|}{t^{2}} \mu_{a}^{2} \delta_{i, 1} & \frac{\partial^{2} T_{k}}{\partial x_{i}^{2}}(0) & =0
\end{aligned}
$$

for $i \in\{1, \ldots, n\}$ and $k \in\{2, \ldots, n\}$. Moreover

$$
\frac{\partial j_{-\|a\| e_{1}}}{\partial x_{i}}(0)=j_{\|a\| e_{1}}(0) \frac{(2-n-\sigma)}{2} \frac{\|a\|}{t^{2}} \delta_{i, 1}
$$

and

$$
\frac{\partial^{2} j_{-\|a\| e_{1}}}{\partial x_{i}^{2}}(0)=-j_{\|a\| e_{1}}(0) \frac{(2-n-\sigma)(n+\sigma)}{4 t^{2}} \frac{\|a\|^{2}}{t^{2}} \delta_{i, 1} .
$$

Therefore, putting $\kappa=n+\sigma$, we have

$$
\begin{aligned}
\Delta\left(\tau_{-\|a\| e_{1}} f\right)(0)= & j_{\|a\| e_{1}}(0) \mu_{a}^{2}\left(\Delta f\left(\|a\| e_{1}\right)-\frac{\|a\|^{2}}{t^{2}} \frac{\partial^{2} f}{\partial x_{1}^{2}}\left(\|a\| e_{1}\right)\right. \\
& \left.-(n+\sigma) \frac{\|a\|}{t^{2}} \frac{\partial f}{\partial x_{1}}\left(\|a\| e_{1}\right)+\frac{(2-\kappa) \kappa}{4 t^{2}} f\left(\|a\| e_{1}\right)\right) \\
& -\frac{(2-\kappa) \kappa}{4 t^{2}} f\left(\|a\| e_{1}\right) j_{\|a\| e_{1}}(0) \\
= & j_{\|a\| e_{1}}(0)\left(L_{\sigma, t} f\left(\|a\| e_{1}\right)-\frac{(2-\kappa) \kappa}{4 t^{2}} f\left(\|a\| e_{1}\right)\right) .
\end{aligned}
$$


Now, as the operator $L_{\sigma, t}$ is invariant under the group $\mathrm{O}(n)$ then we obtain

$$
\begin{aligned}
L_{\sigma, t}\left(f \circ R^{-1}\right)\left(\|a\| e_{1}\right)=\left(j_{\|a\| e_{1}}(0)\right)^{-1} \Delta\left(\tau_{-\|a\| e_{1}}(\right. & \left.\left.f \circ R^{-1}\right)\right)(0) \\
& +\frac{(2-\kappa) \kappa}{4 t^{2}} f\left(R^{-1}\|a\| e_{1}\right)
\end{aligned}
$$

which is equivalent to

$$
\left(L_{\sigma, t} f\right)(a)=\left(j_{a}(0)\right)^{-1} \Delta\left(\tau_{-a} f\right)(0)+\frac{(2-n-\sigma)(n+\sigma)}{4 t^{2}} f(a)
$$

by (5) and the equalities $a=R^{-1}\|a\| e_{1}$ and $j_{\|a\| e_{1}}(0)=j_{R^{-1}\|a\| e_{1}}\left(R^{-1} 0\right)$ $=j_{a}(0)$.

Proposition 5. The operator $L_{\sigma, t}$ commutes with $(\sigma, t)$-translations, i.e.,

$$
L_{\sigma, t}\left(\tau_{b} f\right)=\tau_{b}\left(L_{\sigma, t} f\right) \quad f \in C^{2}\left(\mathbb{B}_{t}^{n}\right), \forall b \in \mathbb{B}_{t}^{n} .
$$

Proof: Using (38) we have

$$
\begin{aligned}
L_{\sigma, t}\left(\tau_{b} f\right)(a)= & \left(j_{a}(0)\right)^{-1} \Delta\left(\tau_{-a} \tau_{b} f\right)(0)+\frac{(2-\kappa) \kappa}{4 t^{2}} \tau_{b} f(a) \\
= & \left.\left(j_{a}(0)\right)^{-1} \Delta\left(f((-b) \oplus(a \oplus x)) \tau_{-a} j_{b}(x)\right)\right|_{x=0} \\
& +\frac{(2-\kappa) \kappa}{4 t^{2}} f((-b) \oplus a) j_{b}(a) .
\end{aligned}
$$

Now, since

$$
(-b) \oplus(a \oplus x)=((-b) \oplus a) \oplus \operatorname{gyr}[-b, a] x \quad \text { by }(8)
$$

and

$$
\begin{aligned}
\tau_{-a} j_{b}(x) & =j_{(-a) \oplus b}(x) & & \text { by }(30) \\
& =j_{b \oplus(-a)}(\operatorname{gyr}[b,-a] x) & & \text { by }(10), \\
& =j_{b \oplus(-a)}(\operatorname{gyr}[-b, a] x) & & \text { by }(15)
\end{aligned}
$$

then together with the invariance of $\Delta$ under the group $\mathrm{O}(n),(26)$ and (22) we obtain

$$
\begin{aligned}
& L_{\sigma, t}\left(\tau_{b} f\right)(a)=\left.\left(j_{a}(0)\right)^{-1} \Delta\left(f(((-b) \oplus a) \oplus \operatorname{gyr}[-b, a] x) j_{b \oplus(-a)}(\operatorname{gyr}[-b, a] x)\right)\right|_{x=0} \\
&+\frac{(2-\kappa) \kappa}{4 t^{2}} f((-b) \oplus a) j_{b}(a) \\
&=\left(j_{a}(0)\right)^{-1} \Delta\left(\tau_{-((-b) \oplus a)} f\right)(0)+\frac{(2-\kappa) \kappa}{4 t^{2}} f((-b) \oplus a) j_{b}(a) \\
&=\left(j_{(-b) \oplus a}(0)\right)^{-1} j_{b}(a) \Delta\left(\tau_{-((-b) \oplus a)} f\right)(0)+\frac{(2-\kappa) \kappa}{4 t^{2}} f((-b) \oplus a) j_{b}(a) \\
&=\left(L_{\sigma, t} f\right)((-b) \oplus a) j_{b}(a)=\tau_{b}\left(L_{\sigma, t} f\right)(a) .
\end{aligned}
$$


For studying some $L^{2}$-properties of the invariant Laplacian $L_{\sigma, t}$ and the $(\sigma, t)$ translation we consider the weighted Hilbert space $L^{2}\left(\mathbb{B}_{t}^{n}, \mathrm{~d} \mu_{\sigma, t}\right)$ with

$$
\mathrm{d} \mu_{\sigma, t}(x)=\left(1-\frac{\|x\|^{2}}{t^{2}}\right)^{\frac{\sigma-3}{2}} \mathrm{~d} x
$$

where $\mathrm{d} x$ stands for the Lebesgue measure in $\mathbb{R}^{n}$. For the special case $\sigma=2-n$ we recover the invariant measure associated to the transformations $T_{a}(x)$.

Proposition 6. For $f, g \in L^{2}\left(\mathbb{B}_{t}^{n}, \mathrm{~d} \mu_{\sigma, t}\right)$ and $a \in \mathbb{B}_{t}^{n}$ we have

$$
\int_{\mathbb{B}_{t}^{n}} \tau_{a} f(x) \overline{g(x)} \mathrm{d} \mu_{\sigma, t}(x)=\int_{\mathbb{B}_{t}^{n}} f(x) \overline{\tau_{-a} g(x)} \mathrm{d} \mu_{\sigma, t}(x) .
$$

Proof: By definition we have

$$
I=\int_{\mathbb{B}_{t}^{n}} \tau_{a} f(x) \overline{g(x)} \mathrm{d} \mu_{\sigma, t}(x)=\int_{\mathbb{B}_{t}^{n}} f((-a) \oplus x) j_{a}(x) \overline{g(x)} \mathrm{d} \mu_{\sigma, t}(x) .
$$

Making the change of variables $(-a) \oplus x=z$, which is equivalent by (12) to $x=a \oplus z$, the measure becomes

$$
\begin{array}{r}
\mathrm{d} \mu_{\sigma, t}(a \oplus z)=\left(\frac{1-\frac{\|a\|^{2}}{t^{2}}}{\left(1+\frac{\langle a, z\rangle}{t^{2}}\right)^{2}}\right)^{\frac{n+1}{2}}\left(1-\frac{\|a \oplus z\|^{2}}{t^{2}}\right)^{\frac{\sigma-3}{2}} \mathrm{~d} z \\
=\left(j_{-a}(z)\right)^{2} \mathrm{~d} \mu_{\sigma, t}(z) \quad \text { by }(3),(21) .
\end{array}
$$

Therefore, it follows

$$
\begin{aligned}
I & =\int_{\mathbb{B}_{t}^{n}} f(z) j_{a}(a \oplus z) \overline{g(a \oplus z)}\left(j_{-a}(z)\right)^{2} \mathrm{~d} \mu_{\sigma, t}(z) \\
& =\int_{\mathbb{B}_{t}^{n}} f(z) \overline{g(a \oplus z)} \tau_{-a} j_{a}(z) j_{-a}(z) \mathrm{d} \mu_{\sigma, t}(z) \\
& =\int_{\mathbb{B}_{t}^{n}} f(z) \overline{\tau_{-a} g(z)} \mathrm{d} \mu_{\sigma, t}(z) \quad \text { by }(29) .
\end{aligned}
$$


Corollary 7. For $f, g \in L^{2}\left(\mathbb{B}_{t}^{n}, \mathrm{~d} \mu_{\sigma, t}\right)$ and $a \in \mathbb{B}_{t}^{n}$ we have

i) $\int_{\mathbb{B}_{t}^{n}} \tau_{a} f(x) \mathrm{d} \mu_{\sigma, t}(x)=\int_{\mathbb{B}_{t}^{n}} f(x) j_{-a}(x) \mathrm{d} \mu_{\sigma, t}(x)$

ii) If $\sigma=2-n$ then $\int_{\mathbb{B}_{t}^{n}} \tau_{a} f(x) \mathrm{d} \mu_{\sigma, t}(x)=\int_{\mathbb{B}_{t}^{n}} f(x) \mathrm{d} \mu_{\sigma, t}(x)$

iii) $\left\|\tau_{a} f\right\|_{2}=\|f\|_{2}$.

From Corollary 7 we see that the $(\sigma, t)$-translation $\tau_{a}$ is a unitary operator in $L^{2}\left(\mathbb{B}_{t}^{n}, \mathrm{~d} \mu_{\sigma, t}\right)$ and the measure $\mathrm{d} \mu_{\sigma, t}$ is translation invariant only for the case $\sigma=2-n$.

There is an important relation between the operator $L_{\sigma, t}$ and the measure $\mathrm{d} \mu_{\sigma, t}$. Up to a constant the Laplace-Beltrami operator $L_{\sigma, t}$ corresponds to a weighted Laplace operator on $\mathbb{B}_{t}^{n}$ for the weighted measure $\mathrm{d} \mu_{\sigma, t}$ in the sense defined in [7], Section 3.6. From Theorem 11.5 in [7] we know that the Laplace operator on a weighted manifold is essentially self-adjoint if all geodesics balls are relatively compact. Therefore, $L_{\sigma, t}$ can be extended to a self adjoint operator in $L^{2}\left(\mathbb{B}_{t}^{n}, \mathrm{~d} \mu_{\sigma, t}\right)$.

Proposition 8. The operator $L_{\sigma, t}$ is essentially self-adjoint in $L^{2}\left(\mathbb{B}_{t}^{n}, \mathrm{~d} \mu_{\sigma, t}\right)$.

\section{The $(\sigma, t)$-Convolution}

In this section we define the $(\sigma, t)$-convolution of two functions, we study its properties and we establish the respective Young's inequality and gyroassociative law. In the limit $t \rightarrow+\infty$ both definitions and properties tend to their Euclidean counterparts.

Definition 9. The $(\sigma, t)$-convolution of two measurable functions $f$ and $g$ is given by

$$
(f * g)(x)=\int_{\mathbb{B}_{t}^{n}} f(y) \tau_{x} g(-y) j_{x}(x) \mathrm{d} \mu_{\sigma, t}(y), \quad x \in \mathbb{B}_{t}^{n}
$$


By Proposition 6 we have

$$
\begin{aligned}
(f * g)(x) & =\int_{\mathbb{B}_{t}^{n}} \tau_{-x} f(y) g(-y) j_{x}(x) \mathrm{d} \mu_{\sigma, t}(y) \\
& =\int_{\mathbb{B}_{t}^{n}} f(x \oplus y) j_{-x}(y) g(-y) j_{x}(x) \mathrm{d} \mu_{\sigma, t}(y) \quad \text { by }(20) \\
& =\int_{\mathbb{B}_{t}^{n}} f(x \oplus(-y)) j_{-x}(-y) g(y) j_{x}(x) \mathrm{d} \mu_{\sigma, t}(y) \quad(y \mapsto-y) \\
& =\int_{\mathbb{B}_{t}^{n}} \tau_{x} f(-y) g(y) j_{x}(x) \mathrm{d} \mu_{\sigma, t}(y) \quad \text { by }(22),(20) \\
& =(g * f)(x) .
\end{aligned}
$$

Thus, the $(\sigma, t)$-convolution is commutative. Before we prove that it is well defined for $\sigma<1$ we need the following lemma.

Lemma 10. Let $\sigma<1$. Then

$$
\int_{\mathbb{S}^{n-1}} j_{x}(r \xi) j_{x}(x) \mathrm{d} \sigma(\xi) \leq C_{\sigma}
$$

with

$$
C_{\sigma}=\left\{\begin{array}{cl}
1, & \text { if } \sigma \in]-n, 2-n[ \\
\frac{\Gamma\left(\frac{n}{2}\right)\left(\frac{1-\sigma}{2}\right)}{\Gamma\left(\frac{2+n-\sigma}{4}\right) \Gamma\left(\frac{n-\sigma}{4}\right)}, & \text { if } \sigma \in]-\infty,-n[\cup[2-n, 1[.
\end{array}\right.
$$

Proof: Using (A.2 in Appendix A) we obtain

$$
\int_{\mathbb{S}^{n-1}} j_{x}(r \xi) j_{x}(x) \mathrm{d} \sigma(\xi)={ }_{2} F_{1}\left(\frac{n+\sigma-2}{4}, \frac{n+\sigma}{4} ; \frac{n}{2} ; \frac{r^{2}\|x\|^{2}}{t^{4}}\right) .
$$

Considering the function $g(s)={ }_{2} F_{1}\left(\frac{n+\sigma-2}{4}, \frac{n+\sigma}{4} ; \frac{n}{2} ; s\right)$ and applying (A.6 and A.4 in Appendix A) we get

$$
\begin{aligned}
g^{\prime}(s) & =\frac{(n+\sigma-2)(n+\sigma)}{8 n}{ }_{2} F_{1}\left(\frac{n+\sigma+2}{4}, \frac{n+\sigma+4}{4} ; \frac{n}{2}+1 ; s\right) . \\
& =\underbrace{\frac{(n+\sigma-2)(n+\sigma)}{8 n}}_{(I)}(1-s)^{-\frac{1+\sigma}{2}} \underbrace{{ }_{2} F_{1}\left(\frac{n-\sigma+2}{4}, \frac{n-\sigma}{4} ; \frac{n}{2}+1 ; s\right)}_{(I I)} .
\end{aligned}
$$

Since $\sigma<1$ then the hypergeometric function $(I I)$ is positive for $s>0$, and therefore, positive on the interval $[0,1[$. Studying the sign of $(I)$ we conclude that 
the function $g$ is strictly increasing when $\sigma \in]-\infty,-n] \cup[2-n, 1[$ and strictly decreasing when $\sigma \in]-n, 2-n\left[\right.$. Since $\sigma<1$, then it exists the $\operatorname{limit}_{s \rightarrow 1^{-}} g(s)$ and by (A.3) it is given by

$$
g(1)=\frac{\Gamma\left(\frac{n}{2}\right) \Gamma\left(\frac{1-\sigma}{2}\right)}{\Gamma\left(\frac{2+n-\sigma}{4}\right) \Gamma\left(\frac{n-\sigma}{4}\right)} .
$$

Thus,

$$
g(s) \leq \max \{g(0), g(1)\}=C_{\sigma}
$$

with $g(0)=1$.

Proposition 11. Let $\sigma<1$ and $f, g \in L^{1}\left(\mathbb{B}_{t}^{n}, \mathrm{~d} \mu_{\sigma, t}\right)$. Then

$$
\|f * g\|_{1} \leq C_{\sigma}\|f\|_{1}\|\widetilde{g}\|_{1}
$$

where $\widetilde{g}(r)=\underset{\substack{\xi \in \mathbb{S}^{n-1} \\ y \in \mathbb{B}_{t}^{n}}}{\operatorname{ess} \sup } g(\operatorname{gyr}[y, r \xi] r \xi)$ for any $r \in[0, t[$.

Proof: Using (31), (23), and (41) we have

$$
\begin{aligned}
I & =\int_{\mathbb{B}_{t}^{n}}\left|\tau_{x} g(-y)\right| j_{x}(x) \mathrm{d} \mu_{\sigma, t}(x) \\
& =\int_{\mathbb{B}_{t}^{n}}\left|\tau_{y} g(\operatorname{gyr}[y, x] x)\right| j_{x}(0) j_{y}(y) j_{x}(x) \mathrm{d} \mu_{\sigma, t}(x) \\
& =\int_{\mathbb{B}_{t}^{n}}|g(\operatorname{gyr}[y, x] x)| j_{-y}(x) j_{y}(y) \mathrm{d} \mu_{\sigma, t}(x) .
\end{aligned}
$$

Using polar coordinates $x=r \xi$, with $r \in\left[0, t\left[\right.\right.$ and $\xi \in \mathbb{S}^{n-1}$, and the normalised surface area $\mathrm{d} \sigma(\xi)=\mathrm{d} \xi / A_{n-1}$, with $A_{n-1}$ being the surface area of $\mathbb{S}^{n-1}$ we get $I=A_{n-1} \int_{0}^{t}\left(1-\frac{r^{2}}{t^{2}}\right)^{\frac{\sigma-3}{2}} r^{n-1} \mathrm{~d} r \int_{\mathbb{S}^{n-1}}|g(\operatorname{gyr}[y, r \xi] r \xi)| j_{-y}(r \xi) j_{y}(y) \mathrm{d} \sigma(\xi)$.

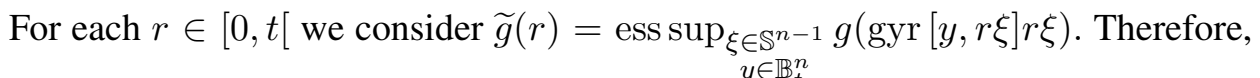
by Lemma 10 and (22) we have we have

$$
\begin{aligned}
I & \leq A_{n-1} \int_{0}^{t}\left(1-\frac{r^{2}}{t^{2}}\right)^{\frac{\sigma-3}{2}} \widetilde{g}(r) r^{n-1} \mathrm{~d} r \int_{\mathbb{S}^{n-1}} j_{-y}(r \xi) j_{-y}(y) \mathrm{d} \sigma(\xi) \\
& \leq C_{\sigma}\|\widetilde{g}\|_{1} .
\end{aligned}
$$


Finally

$$
\begin{aligned}
\|f * g\|_{1} & =\int_{\mathbb{B}_{t}^{n}}\left|\int_{\mathbb{B}_{t}^{n}} f(y) \tau_{x} g(-y) j_{x}(x) \mathrm{d} \mu_{\sigma, t}(y)\right| \mathrm{d} \mu_{\sigma, t}(x) \\
& \leq \int_{\mathbb{B}_{t}^{n}} \int_{\mathbb{B}_{t}^{n}}|f(y)|\left|\tau_{x} g(-y)\right| j_{x}(x) \mathrm{d} \mu_{\sigma, t}(y) \mathrm{d} \mu_{\sigma, t}(x) \\
& =\int_{\mathbb{B}_{t}^{n}}|f(y)|\left(\int_{\mathbb{B}_{t}^{n}}\left|\tau_{x} g(-y)\right| j_{x}(x) \mathrm{d} \mu_{\sigma, t}(x)\right) \mathrm{d} \mu_{\sigma, t}(y) \\
& \leq C_{\sigma}\|f\|_{1}\|\widetilde{g}\|_{1} .
\end{aligned}
$$

In the special case when $g$ is a radial function we obtain as a corollary that $\|f * g\|_{1} \leq C_{\sigma}\|f\|_{1}\|g\|_{1}$ since $\widetilde{g}=g$. We can also prove that for $f \in L^{\infty}\left(\mathbb{B}_{t}^{n}, \mathrm{~d} \mu_{\sigma, t}\right)$ and $g \in L^{1}\left(\mathbb{B}_{t}^{n}, \mathrm{~d} \mu_{\sigma, t}\right)$ we have the inequality

$$
\|f * g\|_{\infty} \leq C_{\sigma}\|\widetilde{g}\|_{1}\|f\|_{\infty}
$$

By (47), (48), and the Riesz-Thorin interpolation Theorem we further obtain for $f \in L^{p}\left(\mathbb{B}_{t}^{n}, \mathrm{~d} \mu_{\sigma, t}\right)$ and $g \in L^{1}\left(\mathbb{B}_{t}^{n}, \mathrm{~d} \mu_{\sigma, t}\right)$ the inequality

$$
\|f * g\|_{p} \leq C_{\sigma}\|\widetilde{g}\|_{1}\|f\|_{p}
$$

To obtain a Young's inequality for the $(\sigma, t)$-convolution we restrict ourselves to the case $\sigma \leq 2-n$.

Theorem 12. Let $\sigma \leq 2-n, 1 \leq p, q, r \leq \infty, \frac{1}{p}+\frac{1}{q}=1+\frac{1}{r}, s=1-\frac{q}{r}$, $f \in L^{p}\left(\mathbb{B}_{t}^{n}, \mathrm{~d} \mu_{\sigma, t}\right)$ and $g \in L^{q}\left(\mathbb{B}_{t}^{n}, \mathrm{~d} \mu_{\sigma, t}\right)$. Then

$$
\|f * g\|_{r} \leq 2^{\frac{2-n-\sigma}{2}}\|\widetilde{g}\|_{q}^{1-s}\|g\|_{q}^{s}\|f\|_{p}
$$

where $\widetilde{g}(x):=\operatorname{ess~sup}_{y \in \mathbb{B}_{t}^{n}} g(\operatorname{gyr}[y, x] x)$, for any $x \in \mathbb{B}_{t}^{n}$.

Proof: First case: $p=1$ and $r=q$. The following estimate is used in the proof

$$
\left.\left.j_{x}(y) j_{x}(x) \leq 2^{\frac{2-n-\sigma}{2}}, \quad x, y \in \mathbb{B}_{t}^{n}, \quad \sigma \in\right]-\infty, 2-n\right] .
$$


Let $K=\|f * g\|_{q}$. Then

$$
\begin{aligned}
& K=\left(\int_{\mathbb{B}_{t}^{n}}\left|\int_{\mathbb{B}_{t}^{n}} f(y) \tau_{x} g(-y) j_{x}(x) \mathrm{d} \mu_{\sigma, t}(y)\right|^{q} \mathrm{~d} \mu_{\sigma, t}(x)\right)^{1 / q} \\
& \leq \int_{\mathbb{B}_{t}^{n}}|f(y)|\left(\int_{\mathbb{B}_{t}^{n}}\left|\tau_{x} g(-y) j_{x}(x)\right|^{q} \mathrm{~d} \mu_{\sigma, t}(x)\right)^{1 / q} \mathrm{~d} \mu_{\sigma, t}(y) \quad \text { (Minkowski's inequality) } \\
& =\int_{\mathbb{B}_{t}^{n}}|f(y)|\left(\int_{\mathbb{B}_{t}^{n}}\left|g(x \oplus(-y)) j_{x}(y) j_{x}(x)\right|^{q} \mathrm{~d} \mu_{\sigma, t}(x)\right)^{1 / q} \mathrm{~d} \mu_{\sigma, t}(y) \quad \text { by }(20) \\
& =\int_{\mathbb{B}_{t}^{n}}|f(y)|\left(\int_{\mathbb{B}_{t}^{n}}\left|g(\operatorname{gyr}[x,-y](-y) \oplus(x)) j_{y}(x) j_{y}(y)\right|^{q} \mathrm{~d} \mu_{\sigma, t}(x)\right)^{1 / q} \mathrm{~d} \mu_{\sigma, t}(y) \\
& \text { by }(10),(24),(23) \\
& =\int_{\mathbb{B}_{t}^{n}}|f(y)|\left(\int_{\mathbb{B}_{t}^{n}}\left|g(\operatorname{gyr}[y \oplus z,-y] z) j_{y}(y \oplus z) j_{y}(y)\right|^{q}\left(j_{y}(-z)\right)^{2} \mathrm{~d} \mu_{\sigma, t}(z)\right)^{1 / q} \mathrm{~d} \mu_{\sigma, t}(y) \\
& \text { (change of variables } z=(-y) \oplus x \text {, and }(40)) \\
& =\int_{\mathbb{B}_{t}^{n}}|f(y)|\left(\int_{\mathbb{B}_{t}^{n}}|g(\operatorname{gyr}[y \oplus z,-y] z)|^{q}\left(j_{y}(y \oplus z) j_{y}(y)\right)^{q-1} j_{y}(-z) j_{y}(y) \mathrm{d} \mu_{\sigma, t}(z)\right)^{1 / q} \mathrm{~d} \mu_{\sigma, t}(y) \\
& \text { by }(29) \\
& \leq 2^{\frac{2-n-\sigma}{2}} \int_{\mathbb{B}_{t}^{n}}|f(y)|\left(\int_{\mathbb{B}_{t}^{n}}|g(\operatorname{gyr}[y, z] z)|^{q} \mathrm{~d} \mu_{\sigma, t}(z)\right)^{1 / q} \mathrm{~d} \mu_{\sigma, t}(y) \quad \text { by (14), (50) } \\
& =2^{\frac{2-n-\sigma}{2}}\|f\|_{1}\|\widetilde{g}\|_{q}
\end{aligned}
$$

Second case: $r=\infty$ and $\frac{1}{p}+\frac{1}{q}=1$. Then

$$
\begin{aligned}
\|f * g\|_{\infty} & =\sup _{x \in \mathbb{B}_{t}^{n}}\left|\int_{\mathbb{B}_{t}^{n}} f(y) \tau_{x} g(-y) j_{x}(x) \mathrm{d} \mu_{\sigma, t}(y)\right| \\
& \leq \sup _{x \in \mathbb{B}_{t}^{n}} \int_{\mathbb{B}_{t}^{n}}\left|f(y) \tau_{x} g(-y) j_{x}(x)\right| \mathrm{d} \mu_{\sigma, t}(y) \\
& \leq \sup _{x \in \mathbb{B}_{t}^{n}}\|f\|_{p}\left(\int_{\mathbb{B}_{t}^{n}}\left|g(x \oplus(-y)) j_{x}(y) j_{x}(x)\right|^{q} \mathrm{~d} \mu_{\sigma, t}(y)\right)^{1 / q}
\end{aligned}
$$




$$
\begin{aligned}
= & \|f\|_{p} \sup _{x \in \mathbb{B}_{t}^{n}}\left(\int_{\mathbb{B}_{t}^{n}}\left|g(z) j_{x}(x \oplus(-z)) j_{x}(x)\right|^{q}\left(j_{x}(z)\right)^{2} \mathrm{~d} \mu_{\sigma, t}(z)\right)^{1 / q} \\
& \text { (change of variables } z=x \oplus(-y), \text { and }(40)) \\
= & \|f\|_{p} \sup _{x \in \mathbb{B}_{t}^{n}}\left(\int_{\mathbb{B}_{t}^{n}}|g(z)|^{q}\left(j_{x}(x \oplus(-z)) j_{x}(x)\right)^{q-1} j_{x}(z) j_{x}(x) \mathrm{d} \mu_{\sigma, t}(z)\right)^{1 / q} \\
& \quad(\text { by }(29)) \\
\leq & 2^{\frac{2-n-\sigma}{2}}\|f\|_{p}\|g\|_{q} .
\end{aligned}
$$

General case: Let $1 \leq q \leq \infty$ and $g \in L^{q}\left(\mathbb{B}_{t}^{n}, \mathrm{~d} \mu_{\sigma, t}\right)$. Considering the linear operator $T$ defined by $T_{g}(f)=f * g$ we have by the previous cases

$$
\left\|T_{g}(f)\right\|_{q} \leq 2^{\frac{2-n-\sigma}{2}}\|\widetilde{g}\|_{q}\|f\|_{1}, \quad \text { i.e., } \quad T: L^{1} \rightarrow L^{q}
$$

and

$$
\left\|T_{g}(f)\right\|_{\infty} \leq 2^{\frac{2-n-\sigma}{2}}\|g\|_{q}\|f\|_{p}, \quad \text { i.e., } \quad T: L^{p} \rightarrow L^{\infty}
$$

with $1 / p+1 / q=1$. By the Riesz-Thorin interpolation theorem we obtain

$$
\left\|T_{g}(f)\right\|_{r} \leq 2^{\frac{2-n-\sigma}{2}}\|\widetilde{g}\|_{q}^{1-s}\|g\|_{q}^{s}\|f\|_{p}
$$

with $\frac{1}{p}+\frac{1}{q}=1+\frac{1}{r}$ and $s=1-\frac{q}{r}$.

Corollary 13. Let $\sigma \leq 2-n, 1 \leq p, q, r \leq \infty, \frac{1}{p}+\frac{1}{q}=1+\frac{1}{r}, f \in L^{p}\left(\mathbb{B}_{t}^{n}, \mathrm{~d} \mu_{\sigma, t}\right)$ and $g \in L^{q}\left(\mathbb{B}_{t}^{n}, \mathrm{~d} \mu_{\sigma, t}\right)$ a radial function. Then

$$
\|f * g\|_{r} \leq 2^{\frac{2-n-\sigma}{2}}\|g\|_{q}\|f\|_{p}
$$

Remark 14. For $\sigma=2-n$ and taking the limit $t \rightarrow+\infty$ in (49) we recover the Young's inequality for the Euclidean convolution in $\mathbb{R}^{n}$ since in the limit $\widetilde{g}=g$.

Another important property of the Euclidean convolution is its translation invariance. Next theorem shows that the $(\sigma, t)$-convolution is gyro-translation invariant.

Theorem 15. The $(\sigma, t)$-convolution is gyro-translation invariant, i.e.,

$$
\tau_{a}(f * g)(x)=\left(\tau_{a} f(\cdot) * g(\operatorname{gyr}[-a, x] \cdot)\right)(x) .
$$

Proof: By (20), (44), and (27) we have

$$
\begin{aligned}
\tau_{a}(f * g)(x) & =(f * g)((-a) \oplus x) j_{a}(x) \\
& =\int_{\mathbb{B}_{t}^{n}} f(y) \tau_{(-a) \oplus x} g(-y) j_{(-a) \oplus x}((-a) \oplus x) j_{a}(x) \mathrm{d} \mu_{\sigma, t}(y) \\
& =\int_{\mathbb{B}_{t}^{n}} f(y) \tau_{(-a) \oplus x} g(-y) j_{x}(x) \mathrm{d} \mu_{\sigma, t}(y) .
\end{aligned}
$$


From (32) we can easily conclude that

$$
\tau_{b \oplus a} f(x)=\tau_{b} \tau_{a} f(\operatorname{gyr}[b, a] x)
$$

since gyr $[a, b] \operatorname{gyr}[b, a]=I$ by (13). Therefore, applying (53) we obtain

$$
\begin{aligned}
\tau_{a}(f * g)(x) & =\int_{\mathbb{B}_{t}^{n}} f(y) \tau_{(-a) \oplus x} g(-y) j_{x}(x) \mathrm{d} \mu_{\sigma, t}(y) \\
& =\int_{\mathbb{B}_{t}^{n}} f(y) \tau_{-a} \tau_{x} g(-\operatorname{gyr}[-a, x] y) j_{x}(x) \mathrm{d} \mu_{\sigma, t}(y) \\
& =\int_{\mathbb{B}_{t}^{n}} \tau_{a} f(y) \tau_{x} g(-\operatorname{gyr}[-a, x] y) j_{x}(x) \mathrm{d} \mu_{\sigma, t}(y) \quad \text { by }(39) \\
& =\left(\tau_{a} f(\cdot) * g(\operatorname{gyr}[-a, x] \cdot)\right)(x) .
\end{aligned}
$$

In Theorem 15 if $g$ is a radial function then we obtain the translation invariant property $\tau_{a}(f * g)=\left(\tau_{a} f\right) * g$. The next theorem shows that the $(\sigma, t)$-convolution is gyroassociative.

Theorem 16. If $f, g, h \in L^{1}\left(\mathbb{B}_{t}^{n}, \mathrm{~d} \mu_{\sigma, t}\right)$ then

$$
\left(f *_{a}\left(g *_{x} h\right)\right)(a)=\left(\left(\left(f(x) *_{y} g(\operatorname{gyr}[a,-(y \oplus x)] \operatorname{gyr}[y, x] x)\right)(y)\right) *_{a} h(y)\right)(a)
$$

Proof: The result of the theorem is proved in the following chain of equations

$$
\begin{aligned}
& (f *(g * h))(a)=\int_{\mathbb{B}_{t}^{n}} f(x) \tau_{a}(h * g)(-x) j_{a}(a) \mathrm{d} \mu_{\sigma, t}(x) \\
& =\int_{\mathbb{B}_{t}^{n}} f(x)(h * g)(a \oplus(-x)) j_{a}(x) j_{a}(a) \mathrm{d} \mu_{\sigma, t}(x) \\
& =\int_{\mathbb{B}_{t}^{n}} f(x) \int_{\mathbb{B}_{t}^{n}} h(y) \tau_{a \oplus(-x)} g(-y) j_{a \oplus(-x)}(a \oplus(-x)) j_{a}(x) \mathrm{d} \mu_{\sigma, t}(y) j_{a}(a) \mathrm{d} \mu_{\sigma, t}(x) \\
& \text { by }(44) \\
& =\int_{\mathbb{B}_{t}^{n}} f(x) \int_{\mathbb{B}_{t}^{n}} h(y) \tau_{a} \tau_{-x} g(-\operatorname{gyr}[a,-x] y) j_{x}(x) \mathrm{d} \mu_{\sigma, t}(y) j_{a}(a) \mathrm{d} \mu_{\sigma, t}(x)
\end{aligned}
$$


$=\int_{\mathbb{B}_{t}^{n}} f(x) \int_{\mathbb{B}_{t}^{n}} \tau_{-a} h(y) \tau_{-x} g(-\operatorname{gyr}[a,-x] y) j_{x}(x) \mathrm{d} \mu_{\sigma, t}(y) j_{a}(a) \mathrm{d} \mu_{\sigma, t}(x)$

by (39)

$=\int_{\mathbb{B}_{t}^{n}} f(x) \int_{\mathbb{B}_{t}^{n}} h(a \oplus y) j_{-a}(y) g(-\operatorname{gyr}[a,-x](x \oplus y)) j_{-x}(y) j_{x}(x) \mathrm{d} \mu_{\sigma, t}(y) j_{a}(a) \mathrm{d} \mu_{\sigma, t}(x)$

$=\int_{\mathbb{B}_{t}^{n}} f(x) \int_{\mathbb{B}_{t}^{n}} \tau_{a} h(-y) \tau_{x} g(\operatorname{gyr}[a,-x] y) j_{x}(x) \mathrm{d} \mu_{\sigma, t}(y) j_{a}(a) \mathrm{d} \mu_{\sigma, t}(x)$

by change $y \mapsto-y,(22),(20)$

$=\int_{\mathbb{B}_{t}^{n}} \tau_{a} h(-y) \int_{\mathbb{B}_{t}^{n}} f(x) \tau_{x} g(\operatorname{gyr}[a,-x] y) j_{x}(x) \mathrm{d} \mu_{\sigma, t}(x) j_{a}(a) \mathrm{d} \mu_{\sigma, t}(y) \quad$ (Fubini)

$=\int_{\mathbb{B}_{t}^{n}} \tau_{a} h(-y) \int_{\mathbb{B}_{t}^{n}} f(x) \tau_{y} g(-\operatorname{gyr}[a,-(y \oplus x)] \operatorname{gyr}[y, x] x) j_{y}(y) \mathrm{d} \mu_{\sigma, t}(x) j_{a}(a) \mathrm{d} \mu_{\sigma, t}(y)$

by $(31),(23)$

$=\left(\left(\left(f(x) *_{y} g(\operatorname{gyr}[a,-(y \oplus x)] \operatorname{gyr}[y, x] x)\right)(y)\right) *_{a} h(y)\right)(a)$.

Corollary 17. If $f, g, h \in L^{1}\left(\mathbb{B}_{t}^{n}, \mathrm{~d} \mu_{\sigma, t}\right)$ and $g$ is a radial function then the $(\sigma, t)$ convolution is associative. i.e.,

$$
f *(g * h)=(f * g) * h .
$$

From Theorem 16 we see that the $(\sigma, t)$-convolution is associative up to a gyration of the argument of the function $g$. However, if $g$ is a radial function then the corresponding gyration is trivial (that is, it is the identity map) and therefore the $(\sigma, t)$-convolution becomes associative. Moreover, in the limit $t \rightarrow+\infty$ gyrations reduce to the identity, so that formula (54) becomes associative in the Euclidean case. If we denote by $L_{R}^{1}\left(\mathbb{B}_{t}^{n}, \mathrm{~d} \mu_{\sigma, t}\right)$ the subspace of $L^{1}\left(\mathbb{B}_{t}^{n}, \mathrm{~d} \mu_{\sigma, t}\right)$ consisting of radial functions then, for $\sigma<1, L_{R}^{1}\left(\mathbb{B}_{t}^{n}, \mathrm{~d} \mu_{\sigma, t}\right)$ is a commutative associative Banach algebra under the $(\sigma, t)$-convolution.

\section{Eigenfunctions of $L_{\sigma, t}$}

Definition 18. For $\lambda \in \mathbb{C}, \xi \in \mathbb{S}^{n-1}$, and $x \in \mathbb{B}_{t}^{n}$ we define the functions $e_{\lambda, \xi ; t}$ by

$$
e_{\lambda, \xi ; t}(x)=\frac{\left(\sqrt{1-\frac{\|x\|^{2}}{t^{2}}}\right)^{\frac{1-\sigma}{2}+\mathrm{i} \lambda t}}{\left(1-\frac{\langle x, \xi\rangle}{t}\right)^{\frac{n-1}{2}+\mathrm{i} \lambda t}} .
$$


The hyperbolic plane waves $e_{\lambda, \xi ; t}(x)$ converge in the limit $t \rightarrow+\infty$ to the Euclidean plane waves $\mathrm{e}^{\mathrm{i}\langle x, \lambda \xi\rangle}$. Since

$$
e_{\lambda, \xi ; t}(x)=\left(1-\frac{\langle x, \xi\rangle}{t}\right)^{-\frac{n-1}{2}-\mathrm{i} \lambda t}\left(\sqrt{1-\frac{\|x\|^{2}}{t^{2}}}\right)^{\frac{1-\sigma}{2}+\mathrm{i} \lambda t}
$$

then we obtain

$$
\lim _{t \rightarrow+\infty} e_{\lambda, \xi ; t}(x)=\lim _{t \rightarrow+\infty}\left[\left(1-\frac{\langle x, \xi\rangle}{t}\right)^{t}\right]^{-i \lambda}=\mathrm{e}^{\mathrm{i}\langle x, \lambda \xi\rangle} .
$$

In the Euclidean case the translation of the Euclidean plane waves $\mathrm{e}^{\mathrm{i}\langle x, \lambda \xi\rangle}$ decomposes into the product of two plane waves one being a modulation. In the hyperbolic case, the $(\sigma, t)$-translation of (55) factorises also in a modulation and the hyperbolic plane wave but it appears a Möbius transformation acting on $\mathbb{S}^{n-1}$ as the next proposition shows.

Proposition 19. The $(\sigma, t)$-translation of $e_{\lambda, \xi ; t}(x)$ admits the factorisation

$$
\tau_{a} e_{\lambda, \xi ; t}(x)=j_{a}(0) e_{\lambda, \xi ; t}(-a) e_{\lambda, a \oplus \xi ; t}(x) .
$$

Proof: Considering $\xi=T_{-a}(\omega)$ with $\xi, \omega \in \mathbb{S}^{n-1}, a \in \mathbb{B}_{t}^{n}$ we have by (2) and (3)

$$
\begin{aligned}
& \tau_{a} e_{\lambda, \xi ; t}(x)=\frac{\left(\sqrt{1-\frac{\left\|T_{-a}(x)\right\|^{2}}{t^{2}}}\right)^{\frac{1-\sigma}{2}+\mathrm{i} \lambda t}}{\left(1-\frac{\left\langle T_{-a}(x), T_{-a}(\omega)\right\rangle}{t}\right)^{\frac{n-1}{2}+\mathrm{i} \lambda t}}\left(\frac{\sqrt{1-\frac{\|a\|^{2}}{t^{2}}}}{1-\frac{\langle a, x\rangle}{t^{2}}}\right)^{\frac{n+\sigma-2}{2}}\left(\frac{\left(1-\frac{\langle a, x\rangle}{t^{2}}\right)\left(1-\frac{\langle a, \omega\rangle}{t}\right)}{\left(1-\frac{\|a\|^{2}}{t^{2}}\right)\left(1-\frac{\langle x, \omega\rangle}{t}\right)}\right)^{\frac{n-1}{2}+\mathrm{i} \lambda t} \\
&=\left(\frac{\sqrt{\left(1-\frac{\|a\|^{2}}{t^{2}}\right)\left(1-\frac{\|x\|^{2}}{t^{2}}\right)}}{1-\frac{\langle x, a\rangle}{t^{2}}}\right)^{\frac{1-\sigma}{2}+\mathrm{i} \lambda t} \\
&\left.\times\left(\frac{\sqrt{1-\frac{\|a\|^{2}}{t^{2}}}}{1-\frac{\langle a, x\rangle}{t^{2}}}\right)^{\frac{n+\sigma-2}{2}}\right)^{\frac{1-\sigma}{2}+\mathrm{i} \lambda t}\left(1-\frac{\langle a, \omega\rangle}{t}\right. \\
&= \frac{\left(\sqrt{1-\frac{\|x\|^{2}}{t^{2}}}\right)^{\frac{n-1}{2}+\mathrm{i} \lambda t}\left(\sqrt{1-\frac{\|a\|^{2}}{t^{2}}}\right)^{\frac{n-1}{2}+\mathrm{i} \lambda t} .}{\left(1-\frac{\langle x, \omega\rangle}{t}\right)^{\frac{n-1}{2}+\mathrm{i} \lambda t}\left(\frac{\|a\|^{2}}{t^{2}}\right.} .
\end{aligned}
$$


Since $\omega=T_{a}(\xi)=a \oplus \xi$ then considering the analogous formula (4) for $T_{a}(\xi)$

$$
1-\frac{\left\langle a, T_{a}(\xi)\right\rangle}{t}=\frac{1-\frac{\|a\|^{2}}{t^{2}}}{1+\frac{\langle\xi, a\rangle}{t}}
$$

we obtain

$$
\tau_{a} e_{\lambda, \xi ; t}(x)=j_{a}(0) e_{\lambda, \xi ; t}(-a) e_{\lambda, a \oplus \xi ; t}(x)
$$

Remark 20. The fractional linear mappings $T_{a}(\xi)=a \oplus \xi, a \in \mathbb{B}_{t}^{n}, \xi \in \mathbb{S}^{n-1}$ are obtained from (1) making the formal substitutions $\frac{x}{t}=\xi$ and $\frac{T_{a}(x)}{t}=T_{a}(\xi)$ and are given by

$$
T_{a}(\xi)=\frac{\frac{a}{t}+P_{a}(\xi)+\mu_{a} Q_{a}(\xi)}{1+\frac{\langle\xi, a\rangle}{t}} .
$$

They map $\mathbb{S}^{n-1}$ onto itself for any $t>0$ and $a \in \mathbb{B}_{t}^{n}$, and in the limit $t \rightarrow+\infty$ they reduce to the identity mapping on $\mathbb{S}^{n-1}$.

Therefore, formula (57) converges in the limit to the well-known formula in the Euclidean case

$$
\mathrm{e}^{\mathrm{i}\langle-a+x, \lambda \xi\rangle}=\mathrm{e}^{\mathrm{i}\langle-a, \lambda \xi\rangle} e^{\mathrm{i}\langle x, \lambda \xi\rangle}, \quad a, x, \lambda \xi \in \mathbb{R}^{n} .
$$

Proposition 21. The function $e_{\lambda, \xi ; t}$ is an eigenfunction of $L_{\sigma, t}$ with eigenvalue $-\lambda^{2}-\frac{(1-\sigma)^{2}}{4 t^{2}}$.

Proof: Applying $L_{\sigma, t}$ to (57) as a function of $y$ and using Proposition 5 we get

$$
\tau_{-x}\left(L_{\sigma, t} e_{\lambda, \xi ; t}\right)(y)=L_{\sigma, t}\left(\tau_{-x} e_{\lambda, \xi ; t}\right)(y)=e_{\lambda, \xi ; t}(x) L_{\sigma, t} e_{\lambda,(-x) \oplus \xi ; t}(y) j_{-x}(0) .
$$

Putting $y=0$ we have

$$
L_{\sigma, t} e_{\lambda, \xi ; t}(x) j_{-x}(0)=L_{\sigma, t} e_{\lambda,(-x) \oplus \xi ; t}(0) e_{\lambda, \xi ; t}(x) j_{-x}(0) .
$$

Thus, we conclude that $e_{\lambda, \xi ; t}(x)$ is an eigenfunction of $L_{\sigma, t}$ with eigenvalue $L_{\sigma, t} e_{\lambda,(-x) \oplus \xi ; t}(0)$. Computing this value we find that the eigenvalue of $e_{\lambda, \xi ; t}(x)$ is $-\lambda^{2}-\frac{(1-\sigma)^{2}}{4 t^{2}}$.

In the limit $t \rightarrow+\infty$ the eigenvalues of $L_{\sigma, t}$ reduce to the eigenvalues of $\Delta$ in $\mathbb{R}^{n}$. In the Euclidean case given two eigenfunctions $\mathrm{e}^{\mathrm{i}\langle x, \lambda \xi\rangle}$ and $\mathrm{e}^{\mathrm{i}\langle x, \gamma \omega\rangle}, \lambda, \gamma \in \mathbb{R}$, $\xi, \omega \in \mathbb{S}^{n-1}$ of the Laplace operator with eigenvalues $-\lambda^{2}$ and $-\gamma^{2}$ respectively, 
the product of the two eigenfunctions is again an eigenfunction of the Laplace operator with eigenvalue $-\left(\lambda^{2}+\gamma^{2}+2 \lambda \gamma\langle\xi, \omega\rangle\right)$. Indeed

$\Delta\left(\mathrm{e}^{\mathrm{i}\langle x, \lambda \xi\rangle} \mathrm{e}^{\mathrm{i}\langle x, \gamma \omega\rangle}\right)=-\|\lambda \xi+\gamma \omega\|^{2} \mathrm{e}^{\mathrm{i}\langle x, \lambda \xi+\gamma \omega\rangle}=-\left(\lambda^{2}+\gamma^{2}+2 \lambda \gamma\langle\xi, \omega\rangle\right) \mathrm{e}^{\mathrm{i}\langle x, \lambda \xi+\gamma \omega\rangle}$

Unfortunately, in the hyperbolic case this is no longer true in general. The only exception is the case $n=1$ and $\sigma=1$ as the next proposition shows.

Proposition 22. For $n \geq 2$ the product of two eigenfunctions of $L_{\sigma, t}$ is not an eigenfunction of $L_{\sigma, t}$ and for $n=1$ the product of two eigenfunctions of $L_{\sigma, t}$ is an eigenfunction of $L_{\sigma, t}$ only in the case $\sigma=1$.

Proof: Let $e_{\lambda, \xi ; t}$ and $e_{\gamma, \omega ; t}$ be two eigenfunctions of $L_{\sigma, t}$ with eigenvalues $-\lambda^{2}-\frac{(1-\sigma)^{2}}{4 t^{2}}$ and $-\gamma^{2}-\frac{(1-\sigma)^{2}}{4 t^{2}}$ respectively. Since for $n \geq 1$ and $f, g \in C^{2}\left(\mathbb{B}_{t}^{n}\right)$ we have

$$
\begin{aligned}
L_{\sigma, t}(f g)= & \left(L_{\sigma, t} f\right) g+f\left(L_{\sigma, t} g\right)+\left(1-\frac{\|x\|^{2}}{t^{2}}\right)^{2}(2\langle\nabla f, \nabla g\rangle \\
& \left.-\frac{2}{t^{2}}\langle x, \nabla f\rangle\langle x, \nabla g\rangle-\frac{(2-n-\sigma)(n+\sigma)}{4 t^{2}} f g\right)
\end{aligned}
$$

we obtain after straightforward computations

$$
\begin{aligned}
& L_{\sigma, t}\left(e_{\lambda, \xi ; t}(x) e_{\gamma, \omega ; t}(x)\right)=\left[-\lambda^{2}-\gamma^{2}-\frac{(1-\sigma)^{2}}{2 t^{2}}-\left(\frac{(1-\sigma+2 \mathrm{i} \lambda t)(n-1+2 \mathrm{i} \gamma t)}{2 t^{3}}\right.\right. \\
& \left.\times\left(1-\frac{\langle x, \omega\rangle}{t}\right)^{-1}\langle x, \omega\rangle+\frac{(1-\sigma+2 \mathrm{i} \gamma t)(n-1+2 \mathrm{i} \lambda t)}{2 t^{3}}\left(1-\frac{\langle x, \xi\rangle}{t}\right)^{-1}\langle x, \xi\rangle\right) \\
& \times\left(1-\frac{\|x\|^{2}}{t^{2}}\right)+\frac{(1-\sigma+2 \mathrm{i} \lambda t)(1-\sigma+2 \mathrm{i} \gamma t)}{2 t^{2}} \frac{|x|^{2}}{t^{2}}+\frac{(n-1+2 \mathrm{i} \lambda t)(n-1+2 \mathrm{i} \gamma t)}{2 t^{2}} \\
& \left.\times \frac{1-\frac{\|x\|^{2}}{t^{2}}}{\left(1-\frac{\langle x, \xi\rangle}{t}\right)\left(1-\frac{\langle x, \omega\rangle}{t}\right)}\left(\langle\xi, \omega\rangle-\frac{\langle x, \omega\rangle\langle x, \xi\rangle}{t^{2}}\right)-\frac{(\sigma+n)(2-n-\sigma)}{4 t^{2}}\left(1-\frac{\|x\|^{2}}{t^{2}}\right)\right] \\
& \times e_{\lambda, \xi ; t}(x) e_{\gamma, \omega ; t}(x) .
\end{aligned}
$$

Therefore, for $n \geq 2$ and $\sigma \in \mathbb{R}$, the product of two eigenfunctions of $L_{\sigma, t}$ is not an eigenfunction of $L_{\sigma, t}$. For $n=1$ the previous formula reduces to

$$
\begin{aligned}
L_{\sigma, t}\left(e_{\lambda, \xi, t}(x) e_{\gamma, \omega ; t}(x)\right)= & {\left[-\lambda^{2}-\gamma^{2}-2 \lambda \gamma \xi \omega-\frac{1-\sigma}{2 t^{2}}\left(\frac{3-\sigma}{2}\left(1-\frac{\|x\|^{2}}{t^{2}}\right)\right.\right.} \\
& +2 \mathrm{i} x(\gamma \omega+\lambda \xi))] e_{\lambda, \xi ; t}(x) e_{\gamma, \omega ; t}(x) .
\end{aligned}
$$


For $\sigma=1$, which corresponds to the case $\sigma=2-n$ for $n=1$, we further obtain

$$
L_{\sigma, t}\left(e_{\lambda, \xi ; t}(x) e_{\gamma, \omega ; t}(x)\right)=-\left(\lambda^{2}+\gamma^{2}+2 \lambda \gamma \xi \omega\right) e_{\lambda, \xi ; t}(x) e_{\gamma, \omega ; t}(x) .
$$

Therefore, only in the case $n=1$ and $\sigma=1$ the product of two eigenfunctions of $L_{\sigma, t}$ is an eigenfunction of $L_{\sigma, t}$.

As expected, in the limit $t \rightarrow+\infty$, formula (59) converges to (58). In the case when $n=1$ and $\sigma=1$ the hyperbolic plane waves (55) are independent of $\xi$ since they reduce to

$$
e_{\lambda ; t}(x)=\left(\frac{1+\frac{x}{t}}{1-\frac{x}{t}}\right)^{\frac{\mathrm{i} \lambda t}{2}}
$$

and, therefore, the exponential law is valid, i.e., $e_{\lambda ; t}(x) e_{\gamma ; t}(x)=e_{\lambda+\gamma ; t}(x)$. This explains the special case obtained in Proposition 22.

Now we study the radial eigenfunctions of $L_{\sigma, t}$, which are called $(\sigma, t)$-spherical functions.

Definition 23. For each $\lambda \in \mathbb{C}$, we define the elementary $(\sigma, t)$-spherical function $\phi_{\lambda ; t} b y$

$$
\phi_{\lambda ; t}(x)=\int_{\mathbb{S}^{n-1}} e_{\lambda, \xi ; t}(x) \mathrm{d} \sigma(\xi), \quad x \in \mathbb{B}_{t}^{n} .
$$

Using (A.2 in Appendix A) and then (A.4 in Appendix A) we can write this function as

$$
\begin{aligned}
\phi_{\lambda ; t}(x) & =\left(1-\frac{\|x\|^{2}}{t^{2}}\right)^{\frac{1-\sigma+2 \mathrm{i} \lambda t}{4}}{ }_{2} F_{1}\left(\frac{n-1+2 \mathrm{i} \lambda t}{4}, \frac{n+1+2 \mathrm{i} \lambda t}{4} ; \frac{n}{2} ; \frac{\|x\|^{2}}{t^{2}}\right) \\
& =\left(1-\frac{\|x\|^{2}}{t^{2}}\right)^{\frac{1-\sigma-2 \mathrm{i} \lambda t}{4}}{ }_{2} F_{1}\left(\frac{n+1-2 \mathrm{i} \lambda t}{4}, \frac{n-1-2 \mathrm{i} \lambda t}{4} ; \frac{n}{2} ; \frac{\|x\|^{2}}{t^{2}}\right) .
\end{aligned}
$$

Therefore, $\phi_{\lambda ; t}$ is a radial function that satisfies $\phi_{\lambda ; t}=\phi_{-\lambda ; t}$ i.e., $\phi_{\lambda ; t}$ is an even function of $\lambda \in \mathbb{C}$. Putting $\|x\|=t$ tanh $s$, with $s \in \mathbb{R}^{+}$, we have the following relation between $\phi_{\lambda ; t}$ and the Jacobi functions $\varphi_{\lambda t}$ (see B.2 in Appendix B)

$$
\begin{aligned}
\phi_{\lambda ; t}(t \tanh s) & =(\cosh s)^{\frac{n-2+\sigma}{2}}{ }_{2} F_{1}\left(\frac{n-1+2 \mathrm{i} \lambda t}{4}, \frac{n-1-2 \mathrm{i} \lambda t}{4} ; \frac{n}{2} ;-\sinh ^{2}(s)\right) \\
& =(\cosh s)^{\frac{n-2+\sigma}{2}} \varphi_{\lambda t}^{\left(\frac{n}{2}-1,-\frac{1}{2}\right)}(s) .
\end{aligned}
$$

The following theorem characterises all $(\sigma, t)$-spherical functions. 
Theorem 24. The function $\phi_{\lambda ; t}$ is a $(\sigma, t)$-spherical function with eigenvalue $-\lambda^{2}-\frac{(1-\sigma)^{2}}{4 t^{2}}$. Moreover, if we normalize $(\sigma, t)$-spherical functions $\phi_{\lambda ; t}$ such that $\phi_{\lambda ; t}(0)=1$, then all $(\sigma, t)$-spherical functions are given by $\phi_{\lambda ; t}$.

Proof: By Proposition 21 it is easy to see that $\phi_{\lambda ; t}$ is an eigenfunction of $L_{\sigma, t}$ with eigenvalue $-\lambda^{2}-\frac{(1-\sigma)^{2}}{4 t^{2}}$. Moreover, $\phi_{\lambda ; t}(0)=1$. Now let $f$ be a spherical function with eigenvalue $-\lambda^{2}-\frac{(1-\sigma)^{2}}{4 t^{2}}$ and consider

$$
f(x)=\left(1-\frac{\|x\|^{2}}{t^{2}}\right)^{\frac{1-\sigma+2 \mathrm{i} \lambda t}{4}} F\left(\frac{\|x\|^{2}}{t^{2}}\right)
$$

with $F$ a function defined on $\mathbb{B}_{t}^{n}$. Since $f$ is a radial function of the form $f(x)=$ $f_{0}(\|x\|)$ then the operator $L_{\sigma, t}$ can be written as

$$
\begin{aligned}
\left(L_{\sigma, t} f\right)(x) & =\left(1-\frac{\|x\|^{2}}{t^{2}}\right)\left(\left(1-\frac{\|x\|^{2}}{t^{2}}\right) f_{0}^{\prime \prime}(\|x\|)\right. \\
& \left.+f_{0}^{\prime}(\|x\|) \frac{1}{\|x\|}\left(n-1-\frac{n+\sigma}{t^{2}}\|x\|^{2}\right)+\frac{(\sigma+n)(2-n-\sigma)}{4 t^{2}} f_{0}(\|x\|)\right) .
\end{aligned}
$$

Then, considering $\|x\|^{2}=r^{2}$ and after straightforward computations we see that if $f$ given by (63) is an eigenfunction of $L_{\sigma, t}$ then $F$ satisfies the following hypergeometric equation

$$
\begin{aligned}
\frac{r^{2}}{t^{2}}\left(1-\frac{r^{2}}{t^{2}}\right) F^{\prime \prime}\left(\frac{r^{2}}{t^{2}}\right) & +\left(\frac{n}{2}-\left(\frac{n}{2}+\mathrm{i} \lambda t+1\right) \frac{r^{2}}{t^{2}}\right) F^{\prime}\left(\frac{r^{2}}{t^{2}}\right) \\
& -\left(\frac{n-1+2 \mathrm{i} \lambda t}{4}\right)\left(\frac{n+1+2 \mathrm{i} \lambda t}{4}\right) F\left(\frac{r^{2}}{t^{2}}\right)=0 .
\end{aligned}
$$

The smooth solutions at the point 0 of the last equation are multiples of ${ }_{2} F_{1}\left(\frac{n-1+2 \mathrm{i} \lambda t}{4}, \frac{n+1+2 \mathrm{i} \lambda t}{4} ; \frac{n}{2} ; \frac{\|x\|^{2}}{t^{2}}\right)$. Therefore, by (61) $f$ is a constant multiple of $\phi_{\lambda ; t}$.

Now we study the asymptotic behavior of $\phi_{\lambda ; t}$ at infinity. The resulting $c$-function is important for the inversion of the $(\sigma, t)$-Helgason Fourier transform.

Lemma 25. For $\operatorname{Im}(\lambda)<0$ we have

$$
\lim _{s \rightarrow+\infty} \phi_{\lambda ; t}(t \tanh s) \mathrm{e}^{\left(\frac{1-\sigma}{2}-\mathrm{i} \lambda t\right) s}=c(\lambda t)
$$

where $c(\lambda t)$ is the Harish-Chandra c-function given by

$$
c(\lambda t)=\frac{2^{\frac{1-\sigma}{2}-\mathrm{i} \lambda t} \Gamma\left(\frac{n}{2}\right) \Gamma(\mathrm{i} \lambda t)}{\Gamma\left(\frac{n-1+2 \mathrm{i} \lambda t}{4}\right) \Gamma\left(\frac{n+1+2 \mathrm{i} \lambda t}{4}\right)} .
$$


Proof: Considering (62), (B.5 in Appendix B), (B.4 in Appendix B) and the limit $\lim _{s \rightarrow+\infty} \mathrm{e}^{s} / \cosh (s)=2$ we obtain

$$
\begin{aligned}
\lim _{s \rightarrow+\infty} \phi_{\lambda ; t} & (t \tanh s) \mathrm{e}^{\left(\frac{1-\sigma}{2}-\mathrm{i} \lambda t\right) s} \\
= & \lim _{s \rightarrow \infty} \mathrm{e}^{\frac{2-n-\sigma}{2} s}(\cosh s)^{\frac{-(2-n-\sigma)}{2}} \varphi_{\lambda t}^{\left(\frac{n}{2}-1,-\frac{1}{2}\right)}(s) e^{\left(-\mathrm{i} \lambda t+\frac{n-1}{2}\right) s} \\
= & 2^{\frac{2-n-\sigma}{2}} c_{\frac{n}{2}-1,-\frac{1}{2}}(\lambda t)=\frac{2^{\frac{1-\sigma}{2}-\mathrm{i} \lambda t} \Gamma\left(\frac{n}{2}\right) \Gamma(\mathrm{i} \lambda t)}{\Gamma\left(\frac{n-1+2 \mathrm{i} \lambda t}{4}\right) \Gamma\left(\frac{n+1+2 \mathrm{i} \lambda t}{4}\right)} .
\end{aligned}
$$

Remark 26. Using the relation $\Gamma(z) \Gamma\left(z+\frac{1}{2}\right)=2^{1-2 z} \sqrt{\pi} \Gamma(2 z)$ we can write

$$
\Gamma\left(\frac{n+1+2 \mathrm{i} \lambda t}{4}\right)=\Gamma\left(\frac{n-1+2 \mathrm{i} \lambda t}{4}+\frac{1}{2}\right)=\frac{2^{1-\frac{n-1+2 \mathrm{i} \lambda t}{2}} \sqrt{\pi} \Gamma\left(\frac{n-1+2 \mathrm{i} \lambda t}{2}\right)}{\Gamma\left(\frac{n-1+2 \mathrm{i} \lambda t}{4}\right)}
$$

and, therefore, (65) simplifies to

$$
c(\lambda t)=\frac{2^{\frac{n-2-\sigma}{2}}}{\sqrt{\pi}} \frac{\Gamma\left(\frac{n}{2}\right) \Gamma(\mathrm{i} \lambda t)}{\Gamma\left(\frac{n-1}{2}+\mathrm{i} \lambda t\right)} .
$$

Finally, we prove the addition formula for the $(\sigma, t)$-spherical functions.

Proposition 27. For every $\lambda \in \mathbb{C}, t \in \mathbb{R}^{+}$, and $x, y \in \mathbb{B}_{t}^{n}$

$$
\begin{aligned}
\tau_{a} \phi_{\lambda ; t}(x) & =j_{a}(0) \int_{\mathbb{S}^{n-1}} e_{-\lambda, \xi ; t}(a) e_{\lambda, \xi ; t}(x) \mathrm{d} \sigma(\xi) \\
& =j_{a}(0) \int_{\mathbb{S}^{n-1}} e_{\lambda, \xi ; t}(a) e_{-\lambda, \xi ; t}(x) \mathrm{d} \sigma(\xi) .
\end{aligned}
$$

Proof: By (57) we have

$$
\begin{aligned}
\tau_{a} \phi_{\lambda ; t}(x) & =\int_{\mathbb{S}^{n-1}} \tau_{a} e_{\lambda, \xi ; t}(x) \mathrm{d} \sigma(\xi) \\
& =j_{a}(0) \int_{\mathbb{S}^{n-1}} e_{\lambda, \xi ; t}(-a) e_{\lambda, a \oplus \xi ; t}(x) \mathrm{d} \sigma(\xi) .
\end{aligned}
$$

Making the change of variables $a \oplus \xi=\xi^{\prime} \Leftrightarrow \xi=(-a) \oplus \xi^{\prime}$ the measure becomes

$$
\mathrm{d} \sigma(\xi)=\left(\frac{1-\frac{\|a\|^{2}}{t^{2}}}{\left(1-\frac{\left\langle a, \xi^{\prime}\right\rangle}{t}\right)^{2}}\right)^{\frac{n-1}{2}} \mathrm{~d} \sigma\left(\xi^{\prime}\right) .
$$


Therefore

$$
\tau_{a} \phi_{\lambda ; t}(x)=j_{a}(0) \int_{\mathbb{S}^{n-1}} e_{\lambda,(-a) \oplus \xi^{\prime} ; t}(-a) e_{\lambda, \xi^{\prime} ; t}(x)\left(\frac{1-\frac{\|a\|^{2}}{t^{2}}}{\left(1-\frac{\left\langle a, \xi^{\prime}\right\rangle}{t}\right)^{2}}\right)^{\frac{n-1}{2}} \mathrm{~d} \sigma\left(\xi^{\prime}\right) .
$$

Since

$$
\begin{aligned}
e_{\lambda,(-a) \oplus \xi^{\prime} ; t}(-a)\left(\frac{1-\frac{\|a\|^{2}}{t^{2}}}{\left(1-\frac{\left\langle a, \xi^{\prime}\right\rangle}{t}\right)^{2}}\right)^{\frac{n-1}{2}} & =\frac{\left(1-\frac{\|a\|^{2}}{t^{2}}\right)^{\frac{1-\sigma+2 \mathrm{i} \lambda t}{4}}}{\left(1-\frac{\left\langle-a,(-a) \oplus \xi^{\prime}\right\rangle}{t}\right)^{\frac{n-1+2 \mathrm{i} \lambda t}{2}}} \frac{\left(1-\frac{\|a\|^{2}}{t^{2}}\right)^{\frac{n-1}{2}}}{\left(1-\frac{\left\langle a, \xi^{\prime}\right\rangle}{t}\right)^{n-1}} \\
& =\frac{\left(1-\frac{\|a\|^{2}}{t^{2}}\right)^{\frac{1-\sigma-2 \mathrm{i} \lambda t}{4}}}{\left(1-\frac{\left\langle a, \xi^{\prime}\right\rangle}{t}\right)^{\frac{n-1-2 \mathrm{i} \lambda t}{2}}} \quad \text { by }(4) \\
& =e_{-\lambda, \xi^{\prime} ; t}(a)
\end{aligned}
$$

we have

$$
\tau_{a} \phi_{\lambda ; t}(x)=j_{a}(0) \int_{\mathbb{S}^{n-1}} e_{-\lambda, \xi^{\prime} ; t}(a) e_{\lambda, \xi^{\prime} ; t}(x) \mathrm{d} \sigma\left(\xi^{\prime}\right) .
$$

The second equality follows from the fact that $\phi_{\lambda ; t}$ is an even function of $\lambda$, i.e., $\phi_{\lambda ; t}=\phi_{-\lambda ; t}$

\section{The $(\sigma, t)$-Poisson transform}

Definition 28. Let $f \in L^{2}\left(\mathbb{S}^{n-1}\right)$. Then the $(\sigma, t)$-Poisson transform is defined by

$$
P_{\lambda, t} f(x)=\int_{\mathbb{S}^{n-1}} f(\xi) e_{\lambda, \xi ; t}(x) \mathrm{d} \sigma(\xi), \quad x \in \mathbb{B}_{t}^{n} .
$$

For a spherical harmonic $Y_{k}$ of degree $k$ we have by (A.1)

$\left(P_{\lambda, t} Y_{k}\right)(x)=C_{k, \nu}\left(1-\frac{\|x\|^{2}}{t^{2}}\right)^{\mu}{ }_{2} F_{1}\left(\frac{\nu+k}{2}, \frac{\nu+k+1}{2} ; k+\frac{n}{2} ; \frac{\|x\|^{2}}{t^{2}}\right) Y_{k}\left(\frac{x}{t}\right)$

with $\nu=\frac{n-1+2 \mathrm{i} \lambda t}{2}, \mu=\frac{1-\sigma+2 \mathrm{i} \lambda t}{4}$, and $C_{k, \nu}=2^{-k} \frac{(\nu)_{k}}{(n / 2)_{k}}$. For $f=\sum_{k=0}^{\infty} a_{k} Y_{k} \in$ $L^{2}\left(\mathbb{S}^{n-1}\right)$ then $P_{\lambda, t} f$ is given by

$$
\sum_{k=0}^{\infty} a_{k} C_{k, \nu}\left(1-\frac{\|x\|^{2}}{t^{2}}\right)^{\mu}{ }_{2} F_{1}\left(\frac{\nu+k}{2}, \frac{\nu+k+1}{2} ; k+\frac{n}{2} ; \frac{\|x\|^{2}}{t^{2}}\right) Y_{k}\left(\frac{x}{t}\right) .
$$


Now we prove a result about the injectivity of the $(\sigma, t)$-Poisson transform which is important later on.

Proposition 29. The Poisson transform $P_{\lambda, t}$ is injective in $L^{2}\left(\mathbb{S}^{n-1}\right)$ if and only if $\lambda \neq \mathrm{i}\left(\frac{2 k+n-1}{2 t}\right)$ for all $k \in \mathbb{Z}_{+}$.

Proof: Let $\lambda=\mathrm{i}\left(\frac{2 k_{0}+n-1}{2 t}\right)$ for some $k_{0} \in \mathbb{Z}^{+}$. Then by (69) we have that $P_{\lambda, t} Y_{k}=0$, for all $k>k_{0}$. Conversely, if $\lambda \neq \mathrm{i}\left(\frac{2 k_{0}+n-1}{2 t}\right)$ for all $k \in \mathbb{Z}_{+}$, then all the coefficients $((n-1+2 \mathrm{i} \lambda) / 2)_{k}$ are not vanishing for $k \in \mathbb{Z}_{+}$. Hence, by (69) we have that $P_{\lambda, t} f=0$ if and only if $f=0$. Thus, $P_{\lambda, t}$ is injective for every $\lambda \neq \mathrm{i}\left(\frac{2 k_{0}+n-1}{2 t}\right), k \in \mathbb{Z}_{+}$.

Corollary 30. Let $\lambda \neq \mathrm{i}\left(\frac{2 k_{0}+n-1}{2 t}\right), k_{0} \in \mathbb{Z}^{+}$. Then the space of functions $\widehat{f}(\lambda, \xi)$ as $f$ ranges over $C_{0}^{\infty}\left(\mathbb{B}_{t}^{n}\right)$ is dense in $L^{2}\left(\mathbb{S}^{n-1}\right)$.

Proof: Let $g \in L^{2}\left(\mathbb{S}^{n-1}\right)$ be such that

$$
\int_{\mathbb{S}^{n-1}} \widehat{f}(\lambda, \xi) g(\xi) \mathrm{d} \sigma(\xi)=0
$$

for all $f \in C_{0}^{\infty}\left(\mathbb{B}_{t}^{n}\right)$. Therefore

$$
\int_{\mathbb{B}_{t}^{n}} f(x)\left(\int_{\mathbb{S}^{n-1}} g(\xi) e_{-\lambda, \xi ; t}(x) \mathrm{d} \sigma(\xi)\right) \mathrm{d} \mu_{\sigma, t}(x)=0
$$

for all $f \in C_{0}^{\infty}\left(\mathbb{B}_{t}^{n}\right)$, which implies that for every $x \in \mathbb{B}_{t}^{n}$

$$
P_{-\lambda, t} g(x)=\int_{\mathbb{S}^{n-1}} g(\xi) e_{-\lambda, \xi ; t}(x) \mathrm{d} \sigma(\xi)=0 .
$$

Finally, by Proposition 29 we have $g=0$.

\section{The $(\sigma, t)$-Helgason Fourier Transform}

Definition 31. For $f \in C_{0}^{\infty}\left(\mathbb{B}_{t}^{n}\right), \lambda \in \mathbb{C}$ and $\xi \in \mathbb{S}^{n-1}$ we define the $(\sigma, t)$ Helgason Fourier transform of $f$ as

$$
\widehat{f}(\lambda, \xi ; t)=\int_{\mathbb{B}_{t}^{n}} f(x) e_{-\lambda, \xi ; t}(x) \mathrm{d} \mu_{\sigma, t}(x) .
$$


Remark 32. If $f$ is a radial function i.e., $f(x)=f(\|x\|)$, then $\widehat{f}(\lambda, \xi ; t)$ is independent of $\xi$ and reduces by (60) to the so called $(\sigma, t)$-spherical transform of $f$ defined by

$$
\widehat{f}(\lambda ; t)=\int_{\mathbb{B}_{t}^{n}} f(x) \phi_{-\lambda ; t}(x) \mathrm{d} \mu_{\sigma, t}(x) .
$$

Moreover, by (56) we recover in the Euclidean limit the usual Fourier transform in $\mathbb{R}^{n}$.

From Propositions 8 and 21 we obtain the following result.

Proposition 33. If $f \in C_{0}^{\infty}\left(\mathbb{B}_{t}^{n}\right)$ then

$$
\widehat{L_{\sigma, t} f}(\lambda, \xi ; t)=-\left(\lambda^{2}+\frac{(1-\sigma)^{2}}{4 t^{2}}\right) \widehat{f}(\lambda, \xi ; t) \text {. }
$$

Now we study the hyperbolic convolution theorem with respect to the $(\sigma, t)$-Helgason Fourier transform. We begin with the following lemma.

Lemma 34. For $a \in \mathbb{B}_{t}^{n}$ and $f \in C_{0}^{\infty}\left(\mathbb{B}_{t}^{n}\right)$

$$
\widehat{\tau_{a} f}(\lambda, \xi ; t)=j_{a}(0) e_{-\lambda, \xi ; t}(a) \widehat{f}(\lambda,(-a) \oplus \xi ; t) .
$$

Proof: By (39), (57) we have

$$
\begin{aligned}
\widehat{\tau_{a} f}(\lambda, \xi ; t) & =\int_{\mathbb{B}_{t}^{n}} \tau_{a} f(x) e_{-\lambda, \xi ; t}(x) \mathrm{d} \mu_{\sigma, t}(x) \\
& =\int_{\mathbb{B}_{t}^{n}} f(x) \tau_{-a} e_{-\lambda, \xi ; t}(x) \mathrm{d} \mu_{\sigma, t}(x) \\
& =j_{a}(0) e_{-\lambda, \xi ; t}(a) \int_{\mathbb{B}_{t}^{n}} f(x) e_{-\lambda,(-a) \oplus \xi ; t}(x) \mathrm{d} \mu_{\sigma, t}(x) \\
& =j_{a}(0) e_{-\lambda, \xi ; t}(a) \widehat{f}(\lambda,(-a) \oplus \xi ; t) .
\end{aligned}
$$

Theorem 35 (Generalised hyperbolic convolution theorem) Let $f, g \in C_{0}^{\infty}\left(\mathbb{B}_{t}^{n}\right)$. Then

$$
\widehat{f * g}(\lambda, \xi)=\int_{\mathbb{B}_{t}^{n}} f(y) e_{-\lambda, \xi ; t}(y) \widehat{\widetilde{g}}_{y}(\lambda,(-y) \oplus \xi ; t) \mathrm{d} \mu_{\sigma, t}(y)
$$

where $\widetilde{g}_{y}(x)=g(\operatorname{gyr}[y, x] x)$. 
Proof: Let $I=\widehat{f * g}(\lambda, \xi)$. We have

$$
\begin{aligned}
I & =\int_{\mathbb{B}_{t}^{n}}\left(\int_{\mathbb{B}_{t}^{n}} f(y) \tau_{x} g(-y) j_{x}(x) \mathrm{d} \mu_{\sigma, t}(y)\right) e_{-\lambda, \xi ; t}(x) \mathrm{d} \mu_{\sigma, t}(x) \\
& =\int_{\mathbb{B}_{t}^{n}} f(y)\left(\int_{\mathbb{B}_{t}^{n}} \tau_{x} g(-y) e_{-\lambda, \xi ; t}(x) j_{x}(x) \mathrm{d} \mu_{\sigma, t}(x)\right) \mathrm{d} \mu_{\sigma, t}(y) \quad \text { (Fubini) } \\
& =\int_{\mathbb{B}_{t}^{n}} f(y)\left(\int_{\mathbb{B}_{t}^{n}} \tau_{y} g(\operatorname{gyr}[x, y] x) e_{-\lambda, \xi ; t}(x) j_{y}(y) \mathrm{d} \mu_{\sigma, t}(x)\right) \mathrm{d} \mu_{\sigma, t}(y) \\
& =\int_{\mathbb{B}_{t}^{n}} f(y) \widehat{\tau_{y} \widetilde{g}_{y}}(\lambda, \xi ; t) j_{y}(y) \mathrm{d} \mu_{\sigma, t}(y) \quad \text { by }(31),(23) \\
& =\int_{\mathbb{B}_{t}^{n}} f(y) e_{-\lambda, \xi ; t}(y) \widehat{\widetilde{g}}_{y}(\lambda,(-y) \oplus \xi ; t) \mathrm{d} \mu_{\sigma, t}(y) \quad \text { by }(74), \quad(23) .
\end{aligned}
$$

Since in the limit $t \rightarrow+\infty$ gyrations reduce to the identity and $(-y) \oplus \xi$ reduces to $\xi$, formula (75) converges in the Euclidean limit to the well-know Convolution Theorem: $\widehat{f * g}=\widehat{f} \cdot \widehat{g}$. By Remark 32 if $g$ is a radial function we obtain the pointwise product of the $(\sigma, t)$-Helgason Fourier transforms.

Corollary 36. Let $f, g \in C_{0}^{\infty}\left(\mathbb{B}_{t}^{n}\right)$ and $g$ radial. Then

$$
\widehat{f * g}(\lambda, \xi ; t)=\widehat{f}(\lambda, \xi ; t) \widehat{g}(\lambda ; t) .
$$

\section{Inversion of the $(\sigma, t)$-Helgason Fourier Transform and Plancherel's Theorem}

We obtain first an inversion formula for the radial case, that is, for the $(\sigma, t)$ spherical transform.

Lemma 37. The $(\sigma, t)$-spherical transform $\mathcal{H}$ can be written as

$$
\mathcal{H}=\mathcal{J}_{\frac{n}{2}-1,-\frac{1}{2}} \circ M_{\sigma}
$$

where $\mathcal{J}_{\frac{n}{2}-1,-\frac{1}{2}}$ is the Jacobi transform (B.1 in Appendix B) with parameters $\alpha=$ $\frac{n}{2}-1$ and $\beta=-\frac{1}{2}$ and

$$
\left(M_{\sigma} f\right)(s):=2^{1-n} A_{n-1} t^{n}(\cosh s)^{\frac{2-n-\sigma}{2}} f(t \tanh s) \text {. }
$$


Proof: Integrating (72) in polar coordinates $x=r \xi$ and making the change of variables $r=t \tanh s$ we obtain

$$
\begin{aligned}
\widehat{f}(\lambda ; t) & =A_{n-1} \int_{0}^{t} f(r) \phi_{-\lambda ; t}(r)\left(1-\frac{r^{2}}{t^{2}}\right)^{\frac{\sigma-3}{2}} r^{n-1} \mathrm{~d} r \\
& =A_{n-1} \int_{0}^{+\infty} f(t \tanh s) \phi_{-\lambda ; t}(t \tanh s)(\cosh s)^{1-\sigma}(\tanh s)^{n-1} t^{n} \mathrm{~d} s .
\end{aligned}
$$

Applying formula (62) yields

$$
\begin{aligned}
\widehat{f}(\lambda ; t) & =2^{1-n} A_{n-1} t^{n} \int_{0}^{+\infty} f(t \tanh s)(\cosh s)^{\frac{2-n-\sigma}{2}} \varphi_{\lambda t}^{\left(\frac{n}{2}-1,-\frac{1}{2}\right)}(s)(2 \sinh s)^{n-1} \mathrm{~d} s \\
& =\left(\mathcal{J}_{\frac{n}{2}-1,-\frac{1}{2}} \circ M_{\sigma} f\right)(\lambda t) .
\end{aligned}
$$

The previous lemma allow us to obtain a Paley-Wiener Theorem for the $(\sigma, t)$ Helgason Fourier transform by using the Paley-Wiener Theorem for the Jacobi transform (Theorem 46 in Appendix B). Let $C_{0, R}^{\infty}\left(\mathbb{B}_{t}^{n}\right)$ denotes the space of all radial $C^{\infty}$ functions on $\mathbb{B}_{t}^{n}$ with compact support and $\mathcal{E}\left(\mathbb{C} \times S^{n-1}\right)$ the space of functions $g(\lambda, \xi)$ on $\mathbb{C} \times \mathbb{S}^{n-1}$, even and holomorphic in $\lambda$ and of uniform exponential type, i.e., there is a positive constant $A_{g}$ such that for all $n \in \mathbb{N}$

$$
\sup _{(\lambda, \xi) \in \mathbb{C} \times \mathbb{S}^{n-1}}|g(\lambda, \xi)|(1+|\lambda|)^{n} \mathrm{e}^{A_{g}|\operatorname{Im}(\lambda)|}<\infty
$$

where $\operatorname{Im}(\lambda)$ denotes the imaginary part of $\lambda$.

Corollary 38 (Paley-Wiener theorem). The $(\sigma, t)$-Helgason Fourier transform is bijective from $C_{0, R}^{\infty}\left(\mathbb{B}_{t}^{n}\right)$ onto $\mathcal{E}\left(\mathcal{C} \times \mathbb{S}^{n-1}\right)$.

In the sequel we denote $C_{n, t, \sigma}=\frac{1}{2^{\sigma} t^{n-1} \pi A_{n-1}}$.

Theorem 39. For all $f \in C_{0, R}^{\infty}\left(\mathbb{B}_{t}^{n}\right)$ we have the inversion formula

$$
f(x)=C_{n, t, \sigma} \int_{0}^{+\infty} \widehat{f}(\lambda ; t) \phi_{\lambda ; t}(x)|c(\lambda t)|^{-2} \mathrm{~d} \lambda .
$$

Proof: Applying formula B.3 in Appendix B for the Jacobi transform and Lemma 6.7 we obtain

$$
\begin{aligned}
M_{\sigma} f(s) & =\frac{1}{2 \pi} \int_{0}^{+\infty} \widehat{f}(\lambda ; t) \varphi_{\lambda t}^{\left(\frac{n}{2}-1,-\frac{1}{2}\right)}(s)\left|c_{\frac{n}{2}-1,-\frac{1}{2}}(\lambda t)\right|^{-2} t \mathrm{~d} \lambda \\
& =\frac{1}{2 \pi} \int_{0}^{+\infty} \widehat{f}(\lambda ; t)(\cosh s)^{\frac{2-n-\sigma}{2}} \phi_{\lambda ; t}(x) \frac{|c(\lambda t)|^{-2}}{2^{-2+n+\sigma}} t \mathrm{~d} \lambda .
\end{aligned}
$$


In the last equality we use (62) and (65). Applying (77) we obtain

$$
f(t \tanh s)=C_{n, t, \sigma} \int_{0}^{+\infty} \widehat{f}(\lambda ; t) \phi_{\lambda ; t}(x)|c(\lambda t)|^{-2} \mathrm{~d} \lambda .
$$

Since $t \tanh s=r$ we obtain the desired result.

Remark 40. The inversion formula (78) can be written as

$$
f(x)=\frac{C_{n, t, \sigma}}{2} \int_{\mathbb{R}} \widehat{f}(\lambda ; t) \phi_{\lambda ; t}(x)|c(\lambda t)|^{-2} \mathrm{~d} \lambda
$$

since the integrand is an even function of $\lambda \in \mathbb{R}$. Note that $f$ is radial and therefore $\widehat{f}(\lambda ; t)$ is an even function of $\lambda, \phi_{\lambda ; t}=\phi_{-\lambda ; t}$, and $|c(-\lambda t)|=|\overline{c(\lambda t)}|=|c(\lambda t)|$, for $\lambda \in \mathbb{R}$.

Now that we have an inversion formula for the radial case we present our main results, the inversion formula for the $(\sigma, t)$-Helgason Fourier transform and the associated Plancherel's Theorem.

Proposition 41. For $f \in C_{0}^{\infty}\left(\mathbb{B}_{t}^{n}\right)$ and $\lambda \in \mathbb{C}$

$$
f * \phi_{\lambda ; t}(x)=\int_{\mathbb{S}^{n-1}} \widehat{f}(\lambda, \xi ; t) e_{\lambda, \xi ; t}(x) \mathrm{d} \sigma(\xi) .
$$

Proof: By formulas (67), (23), Fubini's Theorem and the fact that $\phi_{\lambda, t}$ is a radial function we have

$$
\begin{aligned}
f * \phi_{\lambda ; t}(x) & =\int_{\mathbb{B}} f(y) \tau_{x} \phi_{\lambda ; t}(y) j_{x}(x) \mathrm{d} \mu_{\sigma, t}(y) \\
& =\int_{\mathbb{B}_{t}^{n}} f(y)\left(\int_{\mathbb{S}^{n-1}} e_{\lambda, \xi ; t}(x) e_{-\lambda, \xi ; t}(y) j_{x}(0) j_{x}(x) \mathrm{d} \sigma(\xi)\right) \mathrm{d} \mu_{\sigma, t}(y) \\
& =\int_{\mathbb{S}^{n-1}}\left(\int_{\mathbb{B}_{t}^{n}} f(y) e_{-\lambda, \xi ; t}(y) \mathrm{d} \mu_{\sigma, t}(y)\right) e_{\lambda, \xi ; t}(x) \mathrm{d} \sigma(\xi) \\
& =\int_{\mathbb{S}^{n-1}} \widehat{f}(\lambda, \xi ; t) e_{\lambda, \xi ; t}(x) \mathrm{d} \sigma(\xi) .
\end{aligned}
$$

Theorem 42 (Inversion formula). If $f \in C_{0}^{\infty}\left(\mathbb{B}_{t}^{n}\right)$ then

$$
f(x)=C_{n, t, \sigma} \int_{0}^{+\infty} \int_{\mathbb{S}^{n-1}} \widehat{f}(\lambda, \xi ; t) e_{\lambda, \xi ; t}(x)|c(\lambda t)|^{-2} \mathrm{~d} \sigma(\xi) \mathrm{d} \lambda .
$$


Proof: Given $f \in C_{0}^{\infty}\left(B_{n}^{t}\right)$ and $x, y \in \mathbb{B}_{t}^{n}$ we consider the radial function

$$
f_{x}(y)=\int_{\mathrm{O}(n)} \tau_{K^{-1} x} f(-K y) j_{x}(x) \mathrm{d} K
$$

where $K \in \mathrm{O}(n)$ and $\mathrm{d} K$ is the normalised Haar measure on $\mathrm{O}(n)$. Applying the inversion formula (78) we get

$$
f_{x}(y)=C_{n, t, \sigma} \int_{0}^{+\infty} \widehat{f}_{x}(\lambda ; t) \phi_{\lambda ; t}(y)|c(\lambda t)|^{-2} \mathrm{~d} \lambda .
$$

By (72) and Fubini's Theorem we have

$$
\begin{aligned}
\widehat{f_{x}}(\lambda ; t) & =\int_{\mathbb{B}_{t}^{n}}\left(\int_{\mathrm{O}(n)} \tau_{K^{-1} x} f(-K y) j_{x}(x) \mathrm{d} K\right) \phi_{-\lambda ; t}(y) \mathrm{d} \mu_{\sigma, t}(y) \\
& =\int_{\mathrm{O}(n)}\left(\int_{\mathbb{B}_{t}^{n}} f(x \oplus(-K y)) j_{K^{-1} x}(y) j_{x}(x) \phi_{-\lambda ; t}(y) \mathrm{d} \mu_{\sigma, t}(y)\right) \mathrm{d} K .
\end{aligned}
$$

Considering the change of variables $K y \mapsto z$ we see that the inner integral is independent on $K$. Then we obtain

$$
\widehat{f_{x}}(\lambda ; t)=\int_{\mathbb{B}_{t}^{n}} \tau_{x} f(-z) \phi_{-\lambda ; t}(z) j_{x}(x) \mathrm{d} \mu_{\sigma, t}(z)=\left(f * \phi_{\lambda ; t}\right)(x) .
$$

Since $f(x)=f_{x}(0)$ it follows from (82), (83), and (80) that

$$
\begin{aligned}
f(x) & =C_{n, t, \sigma} \int_{0}^{+\infty} \widehat{f}_{x}(\lambda ; t) \phi_{\lambda ; t}(0)|c(\lambda t)|^{-2} \mathrm{~d} \lambda \\
& =C_{n, t, \sigma} \int_{0}^{+\infty}\left(f * \phi_{\lambda ; t}\right)(x)|c(\lambda t)|^{-2} \mathrm{~d} \lambda \\
& =C_{n, t, \sigma} \int_{0}^{+\infty} \int_{\mathbb{S}^{n-1}} \widehat{f}(\lambda, \xi ; t) e_{\lambda, \xi ; t}(x)|c(\lambda t)|^{-2} \mathrm{~d} \sigma(\xi) \mathrm{d} \lambda .
\end{aligned}
$$

Remark 43. Applying the inversion formula (79) in the proof of Theorem 42 we can write the inversion formula (81) as

$$
f(x)=\frac{C_{n, t, \sigma}}{2} \int_{\mathbb{R}} \int_{\mathbb{S}^{n-1}} \widehat{f}(\lambda, \xi ; t) e_{\lambda, \xi ; t}(x)|c(\lambda t)|^{-2} \mathrm{~d} \sigma(\xi) \mathrm{d} \lambda .
$$


Theorem 44 (Plancherel's theorem). The $(\sigma, t)$-Helgason Fourier transform extends to isometry from $L^{2}\left(\mathbb{B}_{t}^{n}, \mathrm{~d} \mu_{\sigma, t}\right)$ onto $L^{2}\left(\mathbb{R}^{+} \times \mathbb{S}^{n-1}, C_{n, t, \sigma}|c(\lambda t)|^{-2} \mathrm{~d} \lambda \mathrm{d} \sigma\right)$, i.e.,

$$
\int_{\mathbb{B}_{t}^{n}}|f(x)|^{2} \mathrm{~d} \mu_{\sigma, t}(x)=C_{n, t, \sigma} \int_{0}^{+\infty} \int_{\mathbb{S}^{n-1}}|\widehat{f}(\lambda, \xi ; t)|^{2}|c(\lambda t)|^{-2} \mathrm{~d} \sigma(\xi) \mathrm{d} \lambda .
$$

Proof: For $f, g \in C_{0}^{\infty}\left(\mathbb{B}_{t}^{n}\right)$ we obtain Parseval's relation by the inversion formula (81) and Fubini's Theorem

$$
\begin{aligned}
& C_{n, t, \sigma} \int_{0}^{+\infty} \int_{S^{n-1}} \widehat{f}(\lambda, \xi ; t) \overline{\widehat{g}(\lambda, \xi ; t)}|c(\lambda t)|^{-2} \mathrm{~d} \sigma(\xi) \mathrm{d} \lambda \\
& =C_{n, t, \sigma} \int_{0}^{+\infty} \int_{S^{n-1}} \widehat{f}(\lambda, \xi ; t) \int_{\mathbb{B}_{t}^{n}} \overline{g(x)} e_{\lambda, \xi ; t}(x) \mathrm{d} \mu_{\sigma, t}(x)|c(\lambda t)|^{-2} \mathrm{~d} \sigma(\xi) \mathrm{d} \lambda \\
& =\int_{\mathbb{B}_{t}^{n}}\left[C_{n, t, \sigma} \int_{0}^{+\infty} \int_{S^{n-1}} \widehat{f}(\lambda, \xi ; t) e_{\lambda, \xi ; t}(x)|c(\lambda t)|^{-2} \mathrm{~d} \sigma(\xi) \mathrm{d} \lambda\right] \overline{g(x)} \mathrm{d} \mu_{\sigma, t}(x) \\
& =\int_{\mathbb{B}_{t}^{n}} f(x) \overline{g(x)} \mathrm{d} \mu_{\sigma, t}(x) .
\end{aligned}
$$

By taking $f=g$ we obtain (85) for $f \in C_{0}^{\infty}\left(\mathbb{B}_{t}^{n}\right)$ and the result can be extended to $L^{2}\left(\mathbb{B}_{t}^{n}, \mathrm{~d} \mu_{\sigma, t}\right)$ since $C_{0}^{\infty}\left(\mathbb{B}_{t}^{n}\right)$ is dense in $L^{2}\left(\mathbb{B}_{t}^{n}, \mathrm{~d} \mu_{\sigma, t}\right)$. It remains to prove the surjectivity of the $(\sigma, t)$-Helgason Fourier transform. This can be done in a similar way as in ([12], Theorem 6.14) and therefore we omit the details.

Having obtained the main results we now study the limit $t \rightarrow+\infty$ of the previous results. It is anticipated that in the Euclidean limit we recover the usual inversion formula for the Fourier transform and Plancherel's Theorem on $\mathbb{R}^{n}$. To see that this is indeed the case, we observe that from (66)

$$
\frac{1}{|c(\lambda t)|^{2}}=\frac{\left(A_{n-1}\right)^{2}}{\pi^{n-1} 2^{n-\sigma}}\left|\frac{\Gamma\left(\frac{n-1}{2}+\mathrm{i} \lambda t\right)}{\Gamma(\mathrm{i} \lambda t)}\right|^{2}
$$

with $A_{n-1}=\frac{2 \pi^{\frac{n}{2}}}{\Gamma\left(\frac{n}{2}\right)}$ being the surface area of $\mathbb{S}^{n-1}$. Finally, using (86) the $(\sigma, t)$ Helgason inverse Fourier transform (81) simplifies to

$$
\begin{aligned}
f(x) & =\frac{A_{n-1}}{(2 \pi)^{n} t^{n-1}} \int_{0}^{+\infty} \int_{\mathbb{S}^{n-1}} \widehat{f}(\lambda, \xi ; t) e_{\lambda, \xi ; t}(x)\left|\frac{\Gamma\left(\frac{n-1}{2}+\mathrm{i} \lambda t\right)}{\Gamma(\mathrm{i} \lambda t)}\right|^{2} \mathrm{~d} \sigma(\xi) \mathrm{d} \lambda \\
& =\frac{1}{(2 \pi)^{n}} \int_{0}^{+\infty} \int_{\mathbb{S}^{n-1}} \widehat{f}(\lambda, \xi ; t) e_{\lambda, \xi ; t}(x) \frac{\lambda^{n-1}}{N^{(n)}(\lambda t)} \mathrm{d} \xi \mathrm{d} \lambda
\end{aligned}
$$


with

$$
N^{(n)}(\lambda t)=\left|\frac{\Gamma(i \lambda t)}{\Gamma\left(\frac{n-1}{2}+\mathrm{i} \lambda t\right)}\right|^{2}(\lambda t)^{n-1}
$$

Some particular values are $N^{(1)}(\lambda t)=1, N^{(2)}(\lambda t)=\operatorname{coth}(\lambda t), N^{(3)}=1$, and $N^{(4)}(\lambda t)=\frac{(2 \lambda t)^{2} \operatorname{coth}(\pi \lambda t)}{1+(2 \lambda t)^{2}}$. Since $\lim _{t \rightarrow+\infty} N^{(n)}(\lambda t)=1$, for any $n \in \mathbb{N}$ and $\lambda \in \mathbb{R}^{+}$(see [1]), we conclude that in the Euclidean limit the $(\sigma, t)$-Helgason inverse Fourier transform (87) converges to the usual inverse Fourier transform in $\mathbb{R}^{n}$ written in polar coordinates

$$
f(x)=\frac{1}{(2 \pi)^{n}} \int_{0}^{+\infty} \int_{\mathbb{S}^{n-1}} \widehat{f}(\lambda \xi) \mathrm{e}^{\mathrm{i}\langle x, \lambda \xi\rangle} \lambda^{n-1} \mathrm{~d} \xi \mathrm{d} \lambda, \quad x, \lambda \xi \in \mathbb{R}^{n} .
$$

Finally, Plancherel's Theorem (85) can be written as

$$
\int_{\mathbb{B}_{t}^{n}}|f(x)|^{2} \mathrm{~d} \mu_{\sigma, t}(x)=\frac{1}{(2 \pi)^{n}} \int_{0}^{+\infty} \int_{\mathbb{S}^{n-1}}|\widehat{f}(\lambda, \xi)|^{2} \frac{\lambda^{n-1}}{N^{(n)}(\lambda t)} \mathrm{d} \xi \mathrm{d} \lambda
$$

and, therefore, we have an isometry between the spaces $L^{2}\left(\mathbb{B}_{t}^{n}, \mathrm{~d} \mu_{\sigma, t}\right)$ and $L^{2}\left(\mathbb{R}^{+} \times \mathbb{S}^{n-1}, \frac{\lambda^{n-1}}{(2 \pi)^{n} N^{(n)}(\lambda t)} \mathrm{d} \lambda \mathrm{d} \xi\right)$. Applying the limit $t \rightarrow+\infty$ to (88) we recover Plancherel's Theorem in $\mathbb{R}^{n}$

$$
\int_{\mathbb{R}^{n}}|f(x)|^{2} \mathrm{~d} x=\frac{1}{(2 \pi)^{n}} \int_{0}^{+\infty} \int_{\mathbb{S}^{n-1}}|\widehat{f}(\lambda \xi)|^{2} \lambda^{n-1} \mathrm{~d} \xi \mathrm{d} \lambda
$$

\section{Appendices}

\section{A. Spherical Harmonics}

A spherical harmonic of degree $k \geq 0$ denoted by $Y_{k}$ is the restriction to $\mathbb{S}^{n-1}$ of a homogeneous harmonic polynomial in $\mathbb{R}^{n}$. The set of all spherical harmonics of degree $k$ is denoted by $\mathcal{H}_{k}\left(\mathbb{S}^{n-1}\right)$. This space is a finite dimensional subspace of $L^{2}\left(\mathbb{S}^{n-1}\right)$ and we have the direct sum decomposition

$$
L^{2}\left(\mathbb{S}^{n-1}\right)=\bigoplus_{k=0}^{\infty} \mathcal{H}_{k}\left(\mathbb{S}^{n-1}\right)
$$

The following integrals are obtained from the generalisation of Proposition 5.2 in [25]. 
Lemma 45. Let $\nu \in \mathbb{C}, k \in \mathbb{N}_{0}, t \in \mathbb{R}^{+}$, and $Y_{k} \in \mathcal{H}_{k}\left(\mathbb{S}^{n-1}\right)$. Then

$$
\begin{aligned}
\int_{\mathbb{S}^{n-1}}\left(\frac{\sqrt{1-\frac{\|x\|^{2}}{t^{2}}}}{1-\frac{\langle x, \xi\rangle}{t}}\right)^{\nu} Y_{k}(\xi) \mathrm{d} \sigma(\xi)=2^{-k} \frac{(\nu)_{k}}{(n / 2)_{k}}\left(1-\frac{\|x\|^{2}}{t^{2}}\right)^{\frac{\nu}{2}} \\
\quad \times{ }_{2} F_{1}\left(\frac{\nu+k}{2}, \frac{\nu+k+1}{2} ; k+\frac{n}{2} ; \frac{\|x\|^{2}}{t^{2}}\right) Y_{k}\left(\frac{x}{t}\right)
\end{aligned}
$$

where $x \in \mathbb{B}_{t}^{n},(\nu)_{k}$, denotes the Pochhammer symbol, and $\mathrm{d} \sigma$ is the normalised surface measure on $\mathbb{S}^{n-1}$. In particular, when $k=0$, we have

$\int_{\mathbb{S}^{n-1}}\left(\frac{\sqrt{1-\frac{\|x\|^{2}}{t^{2}}}}{1-\frac{\langle x, \xi\rangle}{t}}\right)^{\nu} \mathrm{d} \sigma(\xi)=\left(1-\frac{\|x\|^{2}}{t^{2}}\right)^{\frac{\nu}{2}}{ }_{2} F_{1}\left(\frac{\nu}{2}, \frac{\nu+1}{2} ; \frac{n}{2} ; \frac{\|x\|^{2}}{t^{2}}\right)$.

The Gauss hypergeometric function ${ }_{2} F_{1}$ is an analytic function for $|z|<1$ defined by

$$
{ }_{2} F_{1}(a, b ; c ; z)=\sum_{k=0}^{\infty} \frac{(a)_{k}(b) k}{(c)_{k}} \frac{z^{k}}{k !}
$$

with $c \notin-\mathbb{N}_{0}$. If $\operatorname{Re}(c-a-b)>0$ and $c \notin-\mathbb{N}_{0}$ then exists the limit $\lim _{t \rightarrow 1^{-}} F_{1}(a, b ; c ; t)$ and equals

$$
{ }_{2} F_{1}(a, b ; c ; 1)=\frac{\Gamma(c) \Gamma(c-a-b)}{\Gamma(c-a) \Gamma(c-b)} .
$$

Some useful properties of this function are

$$
\begin{gathered}
{ }_{2} F_{1}(a, b ; c ; z)=(1-z)^{c-a-b}{ }_{2} F_{1}(c-a, c-b ; c ; z) \\
{ }_{2} F_{1}(a, b ; c ; z)=(1-z)^{-a}{ }_{2} F_{1}\left(a, c-b ; c ; \frac{z}{z-1}\right) \\
\frac{\mathrm{d}}{\mathrm{d} z}{ }_{2} F_{1}(a, b ; c ; z)=\frac{a b}{c}{ }_{2} F_{1}(a+1, b+1 ; c+1 ; z) .
\end{gathered}
$$

\section{B. Jacobi Functions}

The classical theory of Jacobi functions involves the parameters $\alpha, \beta, \lambda \in \mathbb{C}$ (see $[10,11])$. Here we introduce the additional parameter $t \in \mathbb{R}^{+}$since we develop 
our hyperbolic harmonic analysis on a ball of arbitrary radius $t$. For $\alpha, \beta, \lambda \in \mathbb{C}$, $t \in \mathbb{R}^{+}$, and $\alpha \neq-1,-2, \ldots$, we define the Jacobi transform as

$$
\mathcal{J}_{\alpha, \beta} g(\lambda t)=\int_{0}^{+\infty} g(r) \varphi_{\lambda t}^{(\alpha, \beta)}(r) \omega_{\alpha, \beta}(r) \mathrm{d} r
$$

for all functions $g$ defined on $\mathbb{R}^{+}$for which the integral (B.1) is well defined. The weight function $\omega_{\alpha, \beta}$ is given by

$$
\omega_{\alpha, \beta}(r)=(2 \sinh (r))^{2 \alpha+1}(2 \cosh (r))^{2 \beta+1}
$$

and the function $\varphi_{\lambda t}^{(\alpha, \beta)}(r)$ denotes the Jacobi function which is defined as the even $C^{\infty}$ function on $\mathbb{R}$ that equals 1 at 0 and satisfies the Jacobi differential equation

$$
\begin{aligned}
\left(\frac{\mathrm{d}^{2}}{\mathrm{~d} r^{2}}+((2 \alpha+1) \operatorname{coth}(r)+(2 \beta+1) \tanh (r)) \frac{\mathrm{d}}{\mathrm{d} r}\right. & \\
& \left.+(\lambda t)^{2}+(\alpha+\beta+1)^{2}\right) \varphi_{\lambda t}^{(\alpha, \beta)}(r)=0 .
\end{aligned}
$$

The function $\varphi_{\lambda t}^{(\alpha, \beta)}(r)$ can be expressed as an hypergeometric function

$$
\varphi_{\lambda t}^{(\alpha, \beta)}(r)={ }_{2} F_{1}\left(\frac{\alpha+\beta+1+i \lambda t}{2}, \frac{\alpha+\beta+1-i \lambda t}{2} ; \alpha+1 ;-\sinh ^{2}(r)\right) .
$$

Since $\varphi_{\lambda t}^{(\alpha, \beta)}$ are even functions of $\lambda t \in \mathbb{C}$ then $\mathcal{J}_{\alpha, \beta} g(\lambda t)$ is an even function of $\lambda t$. Inversion formulas for the Jacobi transform and a Paley-Wiener Theorem are found in [11]. We denote by $C_{0, R}^{\infty}(\mathbb{R})$ the space of even $C^{\infty}$-functions with compact support on $\mathbb{R}$ and $\mathcal{E}$ the space of even and entire functions $g$ for which there are positive constants $A_{g}$ and $C_{g, n}, n=0,1,2, \ldots$, such that for all $\lambda \in \mathbb{C}$ and all $n=0,1,2, \ldots$

$$
|g(\lambda)| \leq C_{g, n}(1+|\lambda|)^{-n} \mathrm{e}^{A_{g}|\operatorname{Im}(\lambda)|}
$$

where $\operatorname{Im}(\lambda)$ denotes the imaginary part of $\lambda$.

Theorem 46 ([11], p.8, Paley-Wiener theorem). For all $\alpha, \beta \in \mathbb{C}$ with $\alpha \neq-1$, $-2, \ldots$ the Jacobi transform is bijective from $C_{0, R}^{\infty}(\mathbb{R})$ onto $\mathcal{E}$.

The Jacobi transform can be inverted under some conditions [11]. Here we only refer to the case which is used in this paper. 
Theorem 47 ([11], p.9) . Let $\alpha, \beta \in \mathbb{R}$ such that $\alpha>-1, \alpha \pm \beta+1 \geq 0$. Then for every $g \in C_{0, R}^{\infty}(\mathbb{R})$ we have

$$
g(r)=\frac{1}{2 \pi} \int_{0}^{+\infty}\left(\mathcal{J}_{\alpha, \beta} g\right)(\lambda t) \varphi_{\lambda t}^{(\alpha, \beta)}(r)\left|c_{\alpha, \beta}(\lambda t)\right|^{-2} t \mathrm{~d} \lambda
$$

where $c_{\alpha, \beta}(\lambda t)$ is the Harish-Chandra c-function associated to $\mathcal{J}_{\alpha, \beta}(\lambda t)$ given by

$$
c_{\alpha, \beta}(\lambda t)=\frac{2^{\alpha+\beta+1-\mathrm{i} \lambda t} \Gamma(\alpha+1) \Gamma(\mathrm{i} \lambda t)}{\Gamma\left(\frac{\alpha+\beta+1+\mathrm{i} \lambda t}{2}\right) \Gamma\left(\frac{\alpha-\beta+1+\mathrm{i} \lambda t}{2}\right)} .
$$

This theorem provides a generalisation of Theorem 2.3 in [11] for arbitrary $t \in \mathbb{R}^{+}$. From [11] and considering $t \in \mathbb{R}^{+}$arbitrary we have the following asymptotic behavior of $\phi_{\lambda t}^{\alpha, \beta}$ for $\operatorname{Im}(\lambda)<0$

$$
\lim _{r \rightarrow+\infty} \varphi_{\lambda t}^{(\alpha, \beta)}(r) \mathrm{e}^{(-\mathrm{i} \lambda t+\alpha+\beta+1) r}=c_{\alpha, \beta}(\lambda t) .
$$

\section{Acknowledgements}

This work was supported by Portuguese funds through the CIDMA - Center for Research and Development in Mathematics and Applications, and the Portuguese Foundation for Science and Technology (FCT - Fundação para a Ciência e a Tecnologia), within project PEst-OE/MAT/UI4106/2014.

\section{References}

[1] Alonso M. Pogosyan G. and Wolf K., Wigner Functions for Curved Spaces I: On Hyperboloids, J. Math. Phys. 7 (2002) 5857-5871.

[2] Boussejra A. and Intissar A., $L^{2}$ - Concrete Spectral Analysis of the Invariant Laplacian $\Delta_{\alpha, \beta}$ in the Unit Complex Ball $B^{n}$, J. Funct. Anal. 160 (1998) 115-140.

[3] Boussejra A. and Zouhair M., Coherent States Quantization of Generalized Bergman Spaces on the Unit Ball of $\mathbb{C}^{n}$ with a New Formula for Their Associated Berezin Transforms, Arxiv preprint:1204.0934.

[4] Ferreira M. and Ren G., Möbius Gyrogroups: A Clifford Algebra Approach, J. Algebra 328 (2011) 230-253.

[5] Ferreira M., Harmonic Analysis on the Möbius Gyrogroup, submitted.

[6] Foguel T. and Ungar A., Gyrogroups and the Decomposition of Groups Into Twisted Subgroups and Subgroups, Pac. J. Math 197 (2001) 1-11. 
[7] Grigor' yan A., Heat Kernel and Analysis on Manifolds, AMS/IP Studies in Advanced Mathematics 47, AMS, Providence 2009.

[8] Helgason S., Groups and Geometric Analysis, Academic Press, Orlando 1984.

[9] Helgason S., Geometric Analysis on Symmetric Spaces, AMS, Providence 1994.

[10] Koornwinder H., A New Proof of a Paley-Wiener Type Theorem for the Jacobi Transform, Ark. Mat. 13 (1975) 145-159.

[11] Koornwinder H., Jacobi Functions and Analysis on Non-Compact Semisimple Groups, In: Special Functions Group Theoretical Aspects and Applications, Reidel, Dordrecht 1984, pp 1-84.

[12] Liu C. and Peng L., Generalized Helgason-Fourier Transforms Associated to Variants of the Laplace-Beltrami Operators on the Unit Ball in $\mathbb{R}^{n}$, Indiana Univ. Math. J. 58 (2009) 1457-1492.

[13] Malzan J., Quantum Mechanics Presented as Harmonic Analysis, Int. J. Theor. Phys. 9 (1974) 305-321.

[14] Ungar A., Thomas Precession and the Parametrization of the Lorentz Transformation Group, Found. Phys. Lett. 1 (1988) 57-89.

[15] Ungar A., Thomas Precession and its Associated Grouplike Structure, Am. J. Phys. 59 (1991) 824-834.

[16] Ungar A., Thomas Precession: Its Underlying Gyrogroup Axioms and Their Use in Hyperbolic Geometry and Relativistic Physics, Found. Phys. 27 (1997) 881-951.

[17] Kasparian, A. and Ungar A., Lie Gyrovector Spaces, J. Geom. Symmettry Phys. 1 (2004) 3-53.

[18] Ungar A., Analytic Hyperbolic Geometry - Mathematical Foundations and Applications, World Scientific, Singapore 2005.

[19] Ungar A., The Proper-Time Lorentz Group Demystified, J. Geom. Symmetry Phys. 4 (2005) 69-95.

[20] Ungar A., Analytic Hyperbolic Geometry and Albert Einstein's Special Theory of Relativity, World Scientific, Singapore 2008.

[21] Ungar A., Barycentric Calculus in Euclidean and Hyperbolic Geometry: A Comparative Introduction, World Scientific, Hackensack 2010.

[22] Ungar A., Hyperbolic Triangle Centers: The Special Relativistic Approach, Springer, New York 2010.

[23] Ungar A., Hyperbolic Geometry, J. Geom. Symmetry Phys. 32 (2013) 61-86.

[24] Ungar A., Analytic Hyperbolic Geometry in N Dimensions: An Introduction, CRC Press, Boca Raton 2015. 
[25] Volchkov V. V. and Volchkov Vit. V., Harmonic Analysis of Mean Periodic Functions on Symmetric Spaces and the Heisenberg Group, Springer, London 2009.

[26] Zhang G., A Weighted Plancherel Formula II. The Case of the Unit Ball, Studia Math. 102 (1992) 103-120.

Milton Ferreira

School of Technology and Management

Polytechnic Institute of Leiria 2411-901 Leiria, PORTUGAL

and

CIDMA - Center for Research and Development

in Mathematics and Applications

Department of Mathematics

University of Aveiro, PORTUGAL

E-mail address: milton.ferreira@ipleiria.pt 